\title{
Contribution of p62/SQSTM1 to PDGF-BB- induced myofibroblast-like phenotypic transition in vascular smooth muscle cells lacking Smpd1 gene
}

Peng Zhang ${ }^{1,2}$, Yinglu Guan ${ }^{2}$, Jiajie Chen ${ }^{2}$, Xiang Li ${ }^{2}$, Bradley K. McConnell $\mathbb{C}^{2}$, Wei Zhou ${ }^{2}$, Krishna M. Boini ${ }^{2}$ and Yang Zhang ${ }^{2}$

\begin{abstract}
Accumulating evidence indicates a critical role of autophagy in regulating vascular smooth muscle cell (SMC) homeostasis in atherogenesis. However, little is known about the modulatory role of autophagy in PDGF-BB-induced $S M C$ transition towards the synthetic phenotype and extracellular matrix remodeling. We recently demonstrated that acid sphingomyelinase (ASM, encoded by Smpd1 gene) controls autophagy maturation in coronary arterial SMCs. Here, we demonstrate that PDGF-BB stimulation causes a myofibroblast-like non-canonical synthetic phenotype

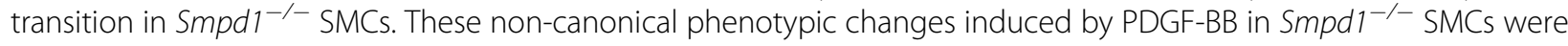
characterized by increased expression of fibroblast-specific protein (FSP-1), massive deposition of collagen type I, decreased cell size, elevated inflammatory status with enhanced cytokine release and adhesion molecule expression. Mechanistically, PDGF-BB induces prolonged Akt activation that causes decreased autophagosome biogenesis and thereby exaggerates p62/SQSTM1 accumulation in Smpd1 $1^{-1-}$ SMCs. More importantly, Akt inhibition or p62/SQSTM1 gene silencing attenuates PDGF-BB-induced phenotypic changes in Smpd1 $1^{-1-}$ SMCs. This first demonstration of a p62/SQSTM1-dependent myofibroblast-like phenotypic transition in Smpd1 ${ }^{-1-}$ SMCs suggests that ASM-mediated autophagy pathway contributes to maintaining the arterial smooth muscle homeostasis in situation of vascular remodeling during atherosclerosis.
\end{abstract}

\section{Introduction}

Vascular smooth muscle cell (SMC) is a highly specialized and differentiated cell and major constitute of blood vessels. SMCs within the adult blood vessel possess contractile phenotype and exhibit a very low synthetic activity. SMCs can switch from a differentiated (contractile) phenotype to a dedifferentiated (synthetic) state that SMCs become proliferative and migratory. The

\footnotetext{
Correspondence: Yang Zhang (yzhan219@central.uh.edu)

'Department of Oncology, Tongji Hospital, Tongji Medical College, Huazhong University of Science and Technology, Wuhan, China

${ }^{2}$ Department of Pharmacological and Pharmaceutical Sciences, College of Pharmacy, University of Houston, Houston, USA
}

Edited by B. Zhivotovsky deregulation of SMC phenotypic plasticity is a pathogenic basis for vascular diseases such as atherosclerosis ${ }^{1}$. In addition to "canonical" synthetic phenotype, SMCs can also switch to other non-canonical phenotypes such as myofibroblast-like phenotypes ${ }^{2}$. Modulation of SMCs toward a myofibroblastic phenotype can occur within the human plaque ${ }^{3}$. It has been proposed that myofibroblastic transition contributes to the formation of atheromatous plaque, a complex inflammatory and fibroproliferative process ${ }^{4}$. Myofibroblasts are cells normally found in pathological situation that are responsible for the production extracellular matrix components (type I and III collagens and fibronectin) as well as inflammatory cytokines at the site of fibrosis ${ }^{5,6}$. Myofibroblasts have 
acquired a phenotype intermediate between fibroblasts and SMCs. They are contractile cells expressing $\alpha$-smooth muscle actin $(\alpha-S M A)$, the actin isoform typical of vascular SMCs, and have a flattened and irregular morphology. In general, myofibroblasts originate from local fibroblasts, however, they are also derived from local SMCs in certain pathological settings. So far, the signaling pathways and mediators through which the SMCs switch to the inflammatory myofibroblasts remain largely undefined.

Acid sphingomyelinase (ASM), encoded by Smpd1 gene, is a lysosome hydrolase that metabolizes sphingomyelin to ceramide and phosphorylcholine ${ }^{7}$. Clinical studies reported that the Niemann-Pick disease patients with deficient ASM activity had high incidences of coronary atherosclerosis ${ }^{8,9}$, suggesting that ASM activity is crucial for preventing atherogenesis in humans. Consistently, adenovirus-mediated ASM expression decreased the lesion formation in atherosclerotic $\mathrm{ApoE}^{-/-}$ mice $^{10}$. In macrophages, ASM-mediated sphingomyelin hydrolysis prevents the retention of cholesterol in lysosomes and foam cell formation ${ }^{11,12}$. Conversely, ASM promotes aggregation and uptake of lipoproteins by arterial-wall macrophages leading foam cell formation $^{13,14}$. It seems ASM participates in various stages of foam cell formation with either anti- or pro-atherogenic roles. Autophagy is a nonstop, reparative, and lifesustaining way to maintain normal cellular homeostasis $^{15}$. Our recent studies demonstrate that ASM is needed for lysosome trafficking and autophagy maturation in vascular SMCs treated with atherogenic oxidized cholesterol $^{16,17}$. ASM exerts its anti-atherogenic effect via modulating autophagy that induces SMCs to a more differentiated contractile phenotype, thereby decreasing cell proliferation and preventing fibrosis ${ }^{18}$. It is intriguing to explore the precise role of ASM and autophagy signaling in modulating myofibroblastic transition in SMCs.

Platelet-derived growth factor-BB (PDGF-BB) is a potent inducer of SMC phenotype switching. The expression of PDGF-BB in vasculature is upregulated in situation of vascular remodeling during atherosclerosis. The present study first identified the role of ASM in SMC myofibroblastic transition by PDGF-BB and then characterized its mechanism of action. As such, we investigated the effects of ASM deficiency by genetic ablation of Smpd1 gene on SMC proliferation, migration, morphological change, extracellular matrix secretion, and inflammatory cytokine production in response to PDGF$\mathrm{BB}$ with in vitro and ex vivo analyses. Moreover, we used recombinant lentiviral vector targeting p62/SQSTM1, a specific autophagy substrate, to test the role of the ASMautophagy-p62/SQSTM1 axis in myofibroblastic transition by PDGF-BB.

\section{Results}

Effects of Smpd1 gene ablation on PDGF-BB-induced phenotypic modulation of SMCs

PDGF-BB is a potent inducer of phenotypic transition of SMCs towards a synthetic phenotype by modulating cell cycle regulators including cyclin $\mathrm{D} 1^{19,20}$. In Smpd $1^{+/+}$ SMCs, PDGF-BB (30 ng/ml) significantly decreased percentage of cells in G0/G1 phase and increased percentage of cells in $S$ phase (Fig. 1a), and increased cyclin D1 (Fig. 1b) and cell numbers (Fig. 1c). These results confirmed that PDGF-BB promoted cell cycle progression and proliferation in Smpd1 $1^{+/+}$SMCs. Smpd1 $1^{-/-}$SMCs exhibited lower level of cyclin D1 and less number of cells in $\mathrm{S}$ phase at the basal condition. However, PDGF-induced changes in cell cycle, cyclin D1 expression, or cell numbers by PDGF-BB were similar in $S m p d 1^{-/-}$and $S m p d 1^{+/+}$ SMCs. These data suggest that Smpd1 gene deletion may affect SMC proliferation at the basal level but is insufficient to inhibit PDGF-BB-induced proliferation.

Next, the effect of Smpd1 gene deficiency on SMC morphology was examined using ECIS technology. As shown in Fig. 1d, the impedance of untreated Smpd $1^{+/+}$ SMCs time-dependently increased to a plateau where cell confluence was achieved. Compared to untreated control, PDGF-BB moderately attenuated the impedance in Smpd $1^{+/+}$SMCs. Similar increase in impedance was seen in untreated Smpd1 ${ }^{-/-}$SMCs, however, PDGF-BB treatment resulted in a marked decrease in impedance in Smpd $1^{-/-}$SMCs (dotted line a and b). The relative changes in the impedance during the first $16 \mathrm{~h}$ were summarized in Fig. 1e. The impedances of untreated Smpd $1^{+/+}$and Smpd1 $1^{-/-}$SMCs increased by $48.20 \pm$ $18.57 \%$ and $27.45 \pm 9.29 \%$, respectively. In the presence of PDGF-BB, the impedance increased by $18.31 \pm 7.03 \%$ in Smpd $1^{+/+}$SMCs, whereas the impedance decreased by $26.22 \pm 7.76 \%$ in Smpd1 $1^{-/-}$SMCs. Because Smpd1 $1^{+/+}$ and $S m p d 1^{-/-}$SMCs exhibit similar proliferation behavior in response to PDGF-BB (Fig. 1a-c), the negative change of the impedance by PDGF-BB suggests that PDGF-BB promotes a morphological change in $S m p d 1^{-/-}$ SMCs leading to decreased cell size. The morphology was further examined by immunofluorescent staining with cytoskeleton protein $\alpha$-SMA and F-actin. Indeed, PDGFBB-treated Smpd1 ${ }^{-/-}$SMCs displayed obviously morphological changes with extended pseudopodia elongation and cell shrinkage (Fig. 1f). SMCs undergo apoptosis show signs of cell shrinkage, round-shaped, and detached. PDGF-BB-treated Smpd1 $1^{-/}$SMCs exhibited extended pseudopodia and remained healthy and proliferative with no obvious increase in detached cells. Thus, the cell shrinkage is unlikely due to apoptosis. In summary, these data suggest that ASM plays a unique role in PDGF-BBinduced morphological changes but not proliferation in SMCs. 
a

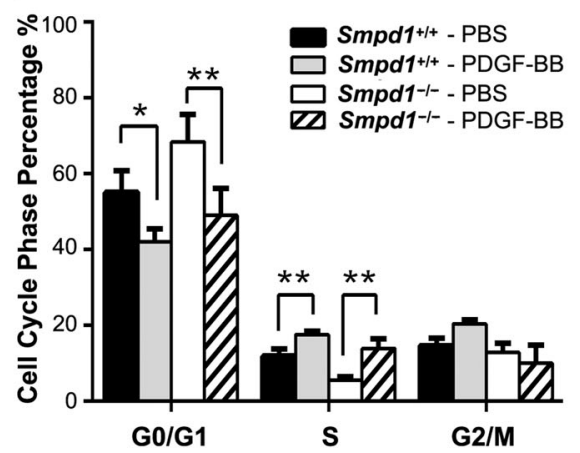

b
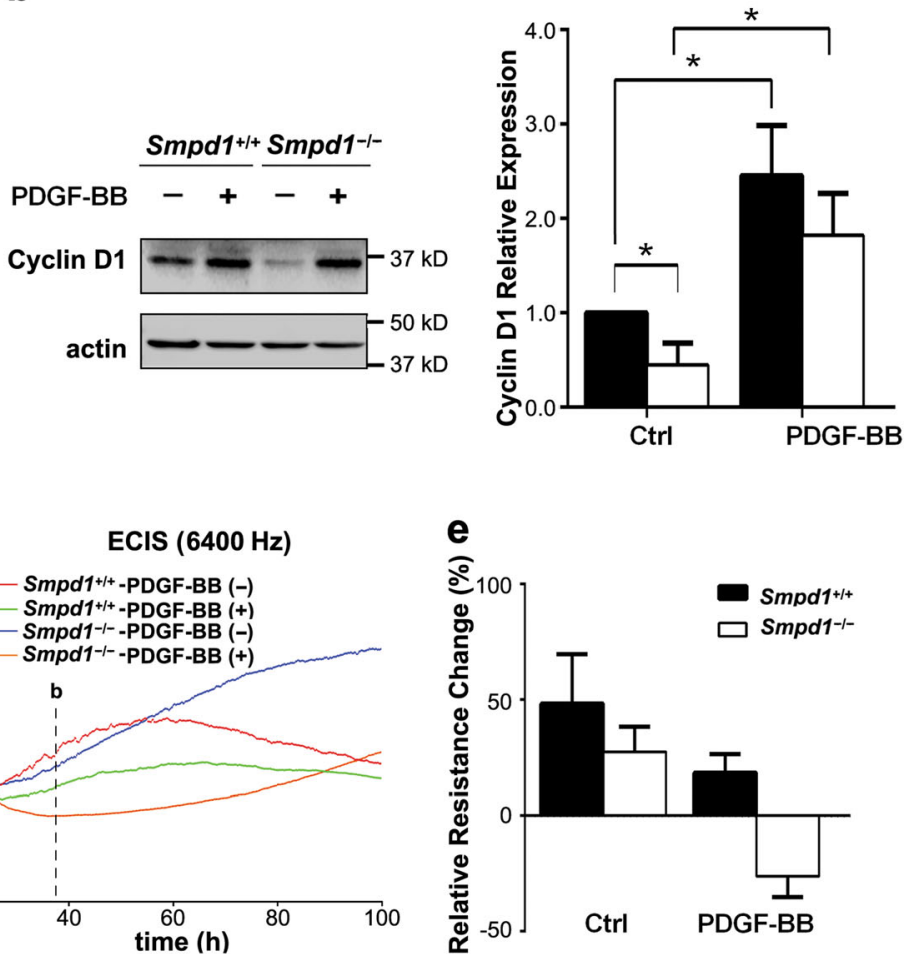

$\operatorname{ECIS}(6400 \mathrm{~Hz})$

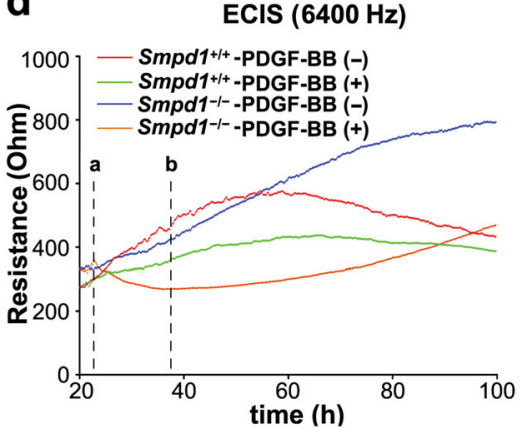

PDGF-BB
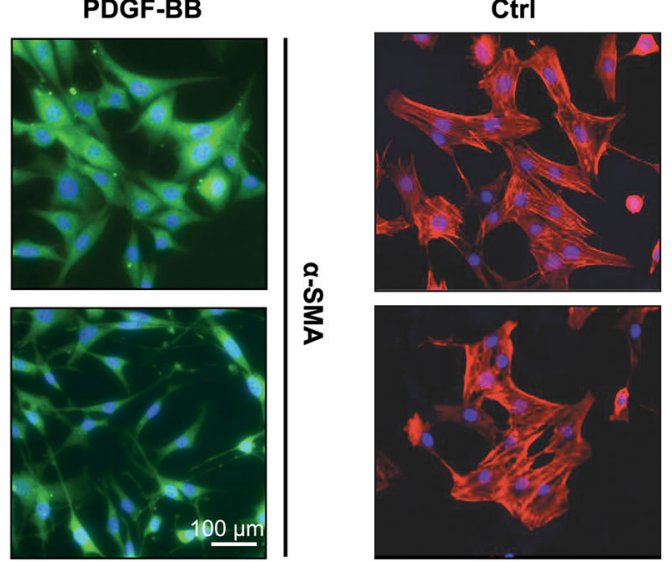

PDGF-BB

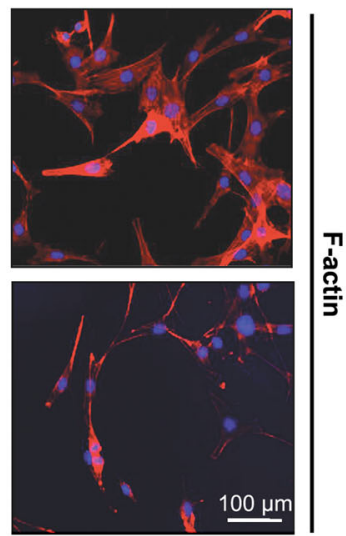

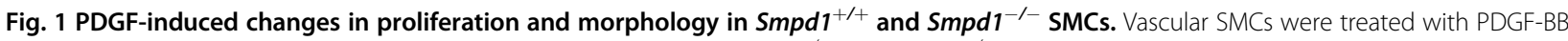

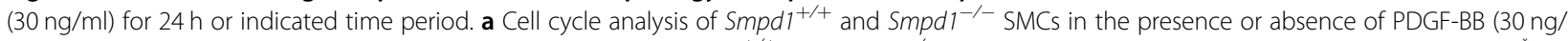
$\mathrm{ml}$ ) by flow cytometry. The bar graph shows the percentage of $S \mathrm{mpd} 1^{+/+}$and $S \mathrm{mpd} 1^{-1-}$ nuclei in the G0/1, S, and G2/M phase of the cell cycle. ${ }^{*} P<$ $0.05,{ }^{* *} P<0.01$ ( $n=4$, Two-way ANOVA). $\mathbf{b}$ Immunoblotting analysis and summarized data show the expression of cyclin D1 in Smpd $1^{+/+}$and Smpd1 $1^{-1}$ SMCs in the presence or absence of PDGF-BB. ${ }^{*} P<0.05\left(n=4\right.$, Two-way ANOVA). c Flow cytometry-based cell counting of Smpd $1^{+/+}$and Smpd $1^{-1-}$ SMCs treated with or with PDGF-BB. ${ }^{*} P<0.05,{ }^{* *} P<0.01(n=4$, Two-way ANOVA). d e ECIS analysis of cell morphology. Cells were seeded

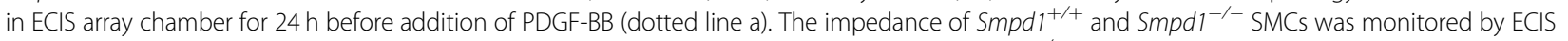
assay within $100 \mathrm{~h}$. The dotted line $\mathrm{b}$ indicates the lowest impedance value of the curve for Smpd1 ${ }^{-1-}$ SMCs treated with PDGF-BB. Summarized data in e show the resistance changes of between dotted line $\mathrm{a}$ and $\mathrm{b}(n=4)$. $\mathbf{f}$ Represent immunofluorescence images for $\mathrm{a}-\mathrm{SMA}$ (green) or F-actin (red) with DAPI (blue) in SMCs. The experiment was repeated at least three times with similar results

Effects of Smpd1 gene ablation on the vascular wall thickness and the formation of aortic sprouts

The association of the morphological changes in Smpd1 $1^{-1-}$ SMCs with vascular wall remodeling was examined in aortic explants from mice (Fig. 2a). The aortic rings were stained with $\alpha$-SMA or Sirus red for collagen. Smpd1 $1^{+/+}$and $S m p d 1^{-/-}$aortic rings appear to be similar in the expression of elastic fiber and vascular 


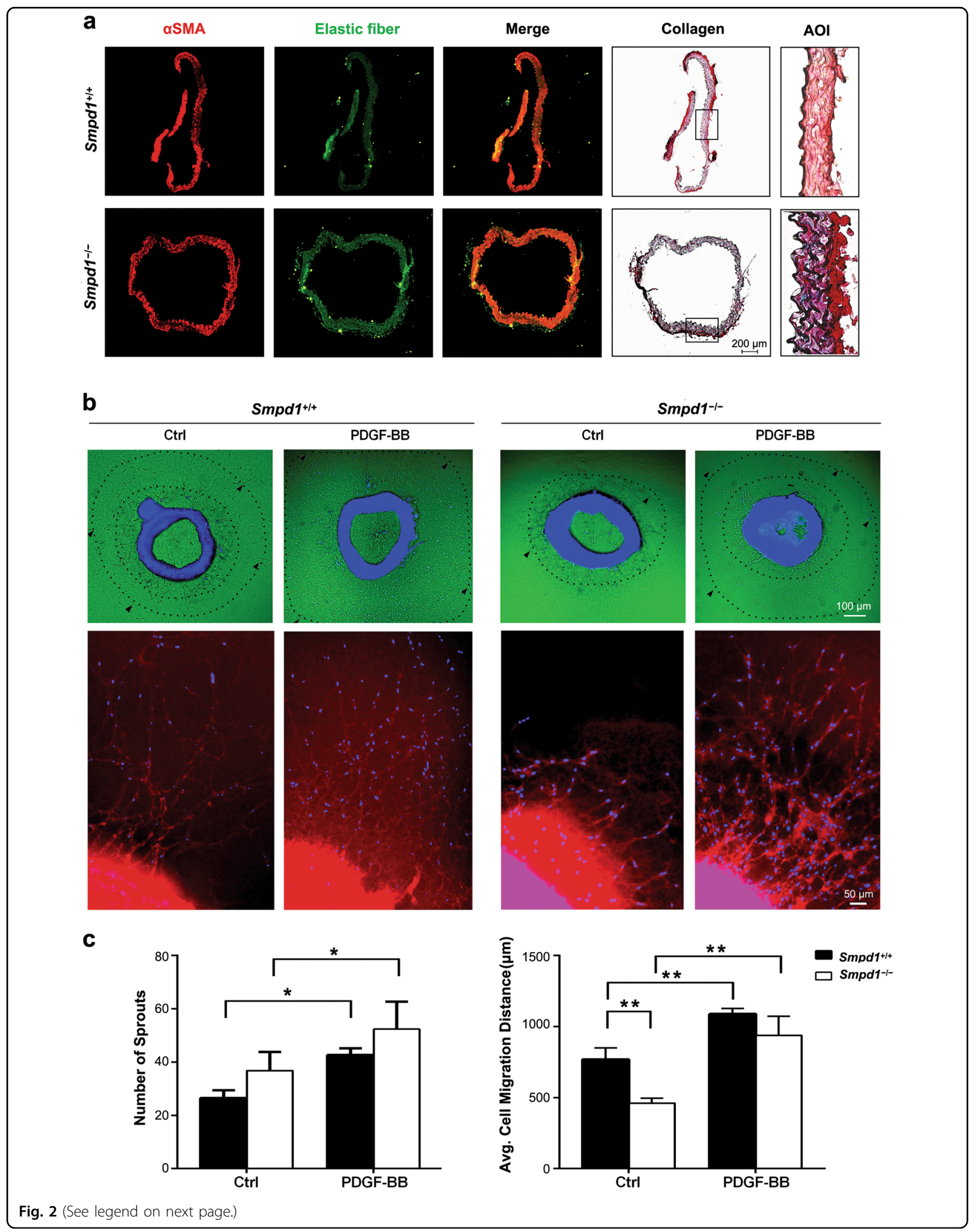


(see figure on previous page)

Fig. 2 Effect of Smpd1 gene ablation on vascular wall thickness and SMC migratory potential. a Immunofluorescence staining for a-SMA (red)

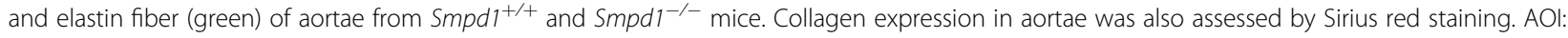
area of interest for enlarged region of Sirius red-collagen staining. b, c Ex vivo aortic sprout analysis of SMC migratory potential. Aortic rings from

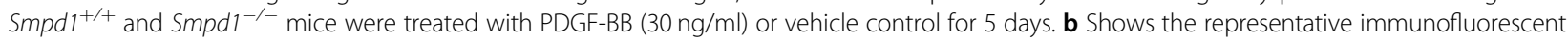
images of aortic ring stained with a-SMA (red) and DAPI (blue). Lower panel $\mathbf{b}$ shows the enlarged images of aortic rings with migrated sprouts. Quantification data in c show the number of SMC sprouts emerging from the aortic ring and the total length of all branches for each outgrowth to reflect migration distance. ${ }^{*} P<0.05(n=6)$

wall thickness. In contrast, lower $\alpha$-SMA expression and higher collagen level (deep red or marron) were observed in $S m p d 1^{-1-}$ rings compared to Smpd $1^{+/+}$ring. These data suggest ASM deficiency leads to enhanced extracellular matrix production.

To determine the role of ASM in migration, the endothelium-denuded aortic rings were cultured ex vivo in Matrigel and treated with PDGF-BB. The number of sprouts that outgrown from aortic rings was quantified by analyzing cells stained with $\alpha$-SMA and DAPI. The cell migration distance was measured for $\alpha-\mathrm{SMA}^{+} / \mathrm{DAPI}^{+}$ cells that emigrate out of aortic explants. As shown in Fig. 2b, untreated Smpd1 $1^{+/+}$aortic rings showed a basal level of sprout outgrowth from aortic explants. Compared to $S m p d 1^{+/+}$rings, untreated $S m p d 1^{-/-}$rings showed significantly decreased cell migration distance but a tendency for an increase in the number of sprouts (Fig. 2c). These data suggest that, at the basal condition, similar number of SMCs can outgrow from Smpd1 $1^{-/}$rings but these $S m p d 1^{-1-}$ SMCs migrate at much reduced speed in Matrigel. However, under PDGF-BB treatment, no significant difference was found in either the number of sprouts or cell migration in $S m p d 1^{+/+}$and $S m p d 1^{-/-}$ rings (Figs. 2b, c), suggesting a maximal activation of migration potential by PDGF-BB in SMCs outgrown from Smpd $1^{+/+}$and Smpd1 $1^{-/-}$rings. Thus, ASM is involved in SMC migration at the basal condition, but it is dispensable for PDGF-BB-induced migration.

\section{PDGF-BB promotes myofibroblast-like phenotype transition of SMCs lacking Smpd1 gene}

The expression of various SMC and fibroblast markers was measured and compared in between $S m p d 1^{-1-}$ and Smpd1 $1^{+/+}$SMCs. As shown in Fig. 3a-c, under basal condition, Smpd1 $1^{-/-}$and Smpd1 $1^{+/+}$SMCs had similar expression level of collagen I and fibroblast-specific protein 1 (FSP1). However, PDGF-BB treatment selectively induced upregulation of collagen I and FSP1 in Smpd1 $1^{-/-}$ SMCs. The collagen I in Smpd1 $1^{-1-}$ cells showed a reduced molecular weight, which may be due to deregulated post-translational modification such as glycosylation $^{21,22}$. In addition, Smpd1 $1^{-/}$SMCs with PDGF-BB also showed different expression pattern of SMC phenotype markers with lower $\alpha$-SMA, higher calponin and SM22, but similar vimentin expression levels. The extracellular collagen deposition was confirmed by staining SMCs with Sirius red. PDGF-BB induced collagen deposition in Smpd1 $1^{+/+}$SMCs, which is more pronounced in Smpd1 $1^{-1-}$ SMCs (Fig. 3d,e). TGF- $\beta 1$ plays a key role in regulating collagen I accumulation in $\mathrm{SMCs}^{23}$. Smpd $1^{-1-}$ cells showed much higher TGF- $\beta 1$ expression under either basal condition or with PDGF-BB stimulation (Fig. 3f). Altogether, these results demonstrate that PDGF-BB potentially promotes the transition to myofibroblast-like phenotype characterized by elevated expression of FSP-1 and massive deposition of collagen type I.

\section{Smpd1 gene ablation exacerbates PDGF-induced inflammation in SMCs}

Myofibroblasts are a major source of inflammation in the diseased organ by recruiting inflammatory cells and secreting inflammatory cytokines. As shown in Fig. 4a, more monocytes were found adhering to Smpd1 $1^{-/-}$ SMCs than Smpd1 $1^{+/+}$SMCs at either the basal level or with PDGF-BB stimulation. Such enhanced recruitment of monocytes to Smpd1 $1^{-/}$SMCs was accompanied by increased expression of intercellular adhesion molecule-1 (ICAM-1) (Fig. 4b, c). As shown in Fig. 4d, Smpd1 $1^{-/-}$ SMCs had higher IL-6 and IL-18 mRNA compared to Smpd $1^{+/+}$SMCs at the basal condition, which was massively enhanced by PDGF-BB. Together, these results provide novel evidence that PDGF-BB-treated Smpd1 $1^{-/-}$ SMCs gain myofibroblast-like phenotype with elevated inflammatory status.

\section{Smpd1 gene ablation increases autophagosome accumulation and p62/SQSTM1 in SMCs}

Accumulating evidence has demonstrated that autophagy is critically involved in SMC phenotypic plasticity ${ }^{18}$. We sought to investigate the role of autophagy in myofibroblast-like transition. First, the dynamic balance of autophagosome formation and its degradation was measured by staining SMCs with CytoID. As shown in Fig. 5a, Smpd1 $1^{-1-}$ SMCs had more autophagic vacuoles (as shown by increased CytoID-positive cells) than Smpd $1^{+/+}$SMCs. This increase was not abolished by chloroquine (CQ), an autophagic flux inhibitor. Smpd1 $1^{-1}$ - SMCs also had higher expression of autophagosome makers including ATG 3, ATG 5, LC3B-II, ATG12 and a 

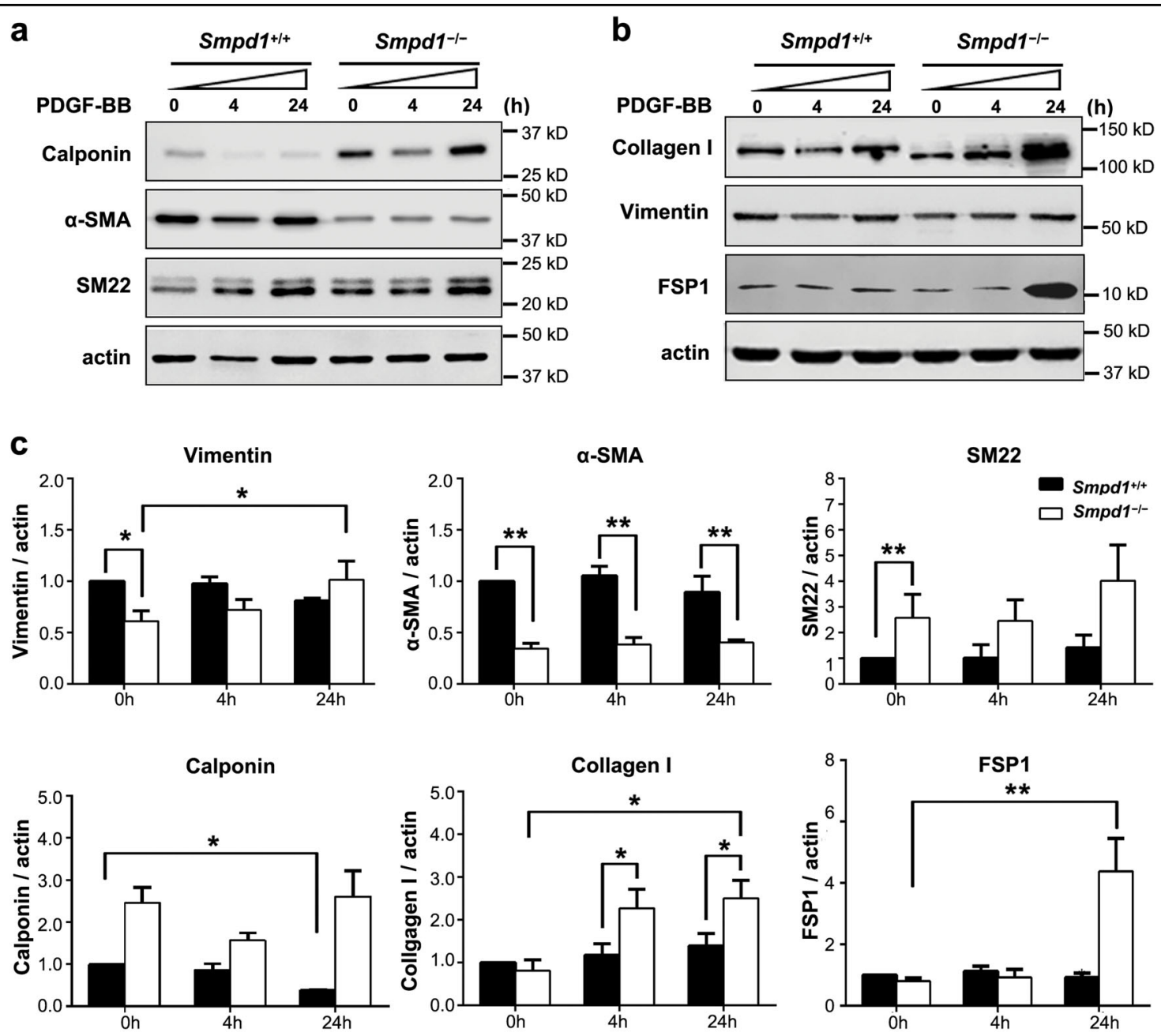

d

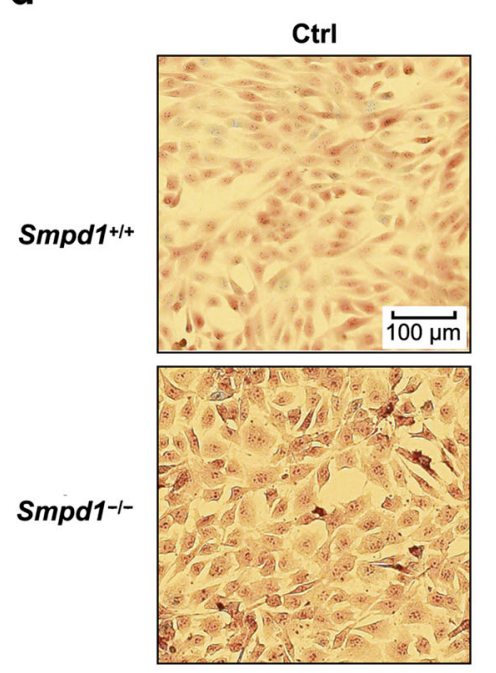

PDGF-BB

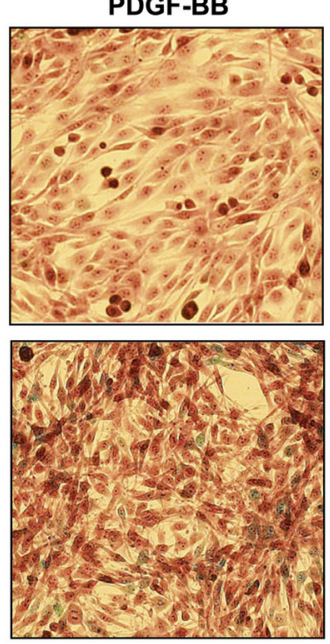

e

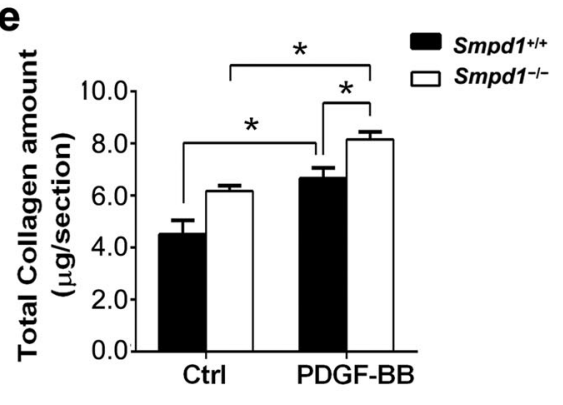

f

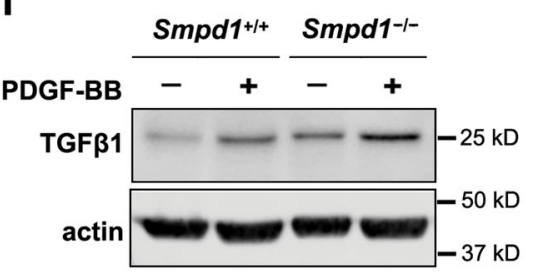

Fig. 3 (See legend on next page.) 
(see figure on previous page)

Fig. 3 PDGF-BB promotes myofibroblast-like differentiation of Smpd $1^{-1-}$ SMCs. SMCs were under control condition or stimulated with PDGF$\mathrm{BB}(30 \mathrm{ng} / \mathrm{ml})$ for the indicated time. a Representative western blot showing the protein expression of SM22, a-SMA, and Calponin. $\mathbf{b}$ Representative immunoblotting analysis of Vimentin, Collagen I, and FSP-1. c Summarized data of immunoblotting mentioned above. ${ }^{*} P<0.05$, ${ }^{* *} P<0.01(n=4)$. d Sirius Red staining of SMCs under control condition or stimulated with PDGF-BB ( $30 \mathrm{ng} / \mathrm{ml})$ for $24 \mathrm{~h}$. e Total collagen amount was examined according to Sirius red staining results. ${ }^{*} P<0.05,{ }^{* *} P<0.01(n=4)$. Two-way ANOVA with genotype and treatment as category factors. f Representative western blot showing the protein expression of TGF- $\beta 1$ in Smpd $1^{+/+}$and Smpd $1^{-1-}$ SMCs before and after PDGF-BB stimulation. The experiment was repeated at least three times with similar results

a
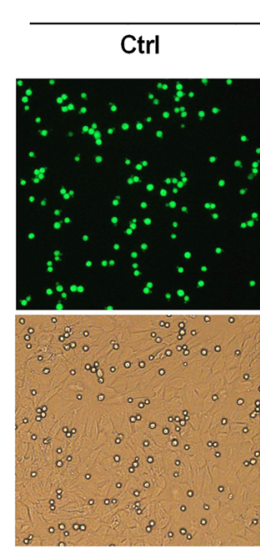

b

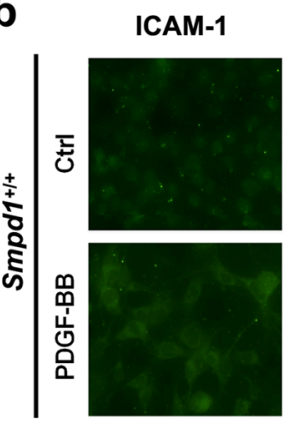

Smpd1+/+

PDGF-BB
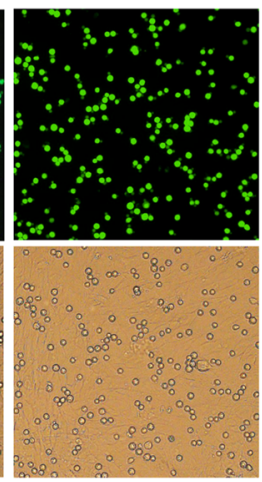
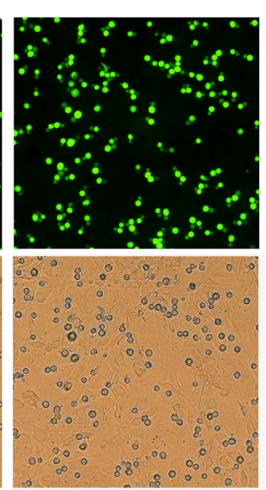

Smpd1-1-
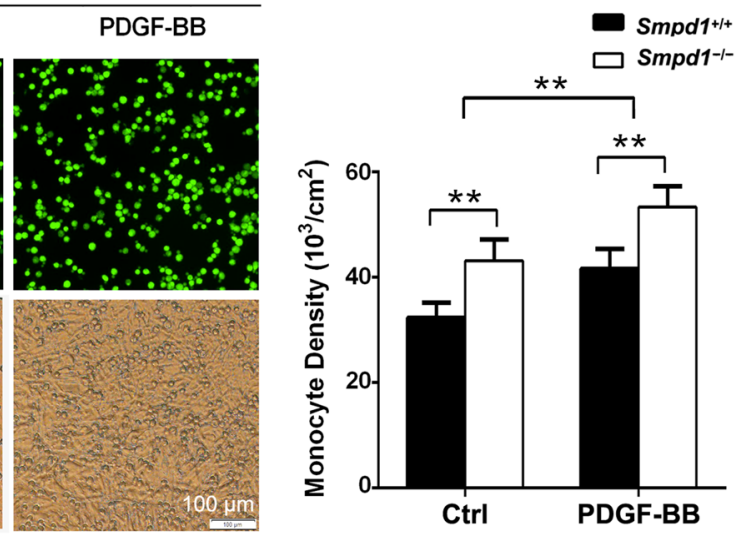

C

ICAM-1
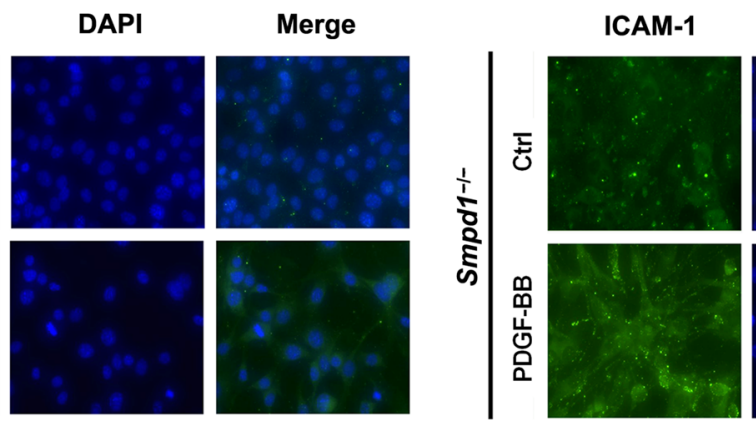

DAPI

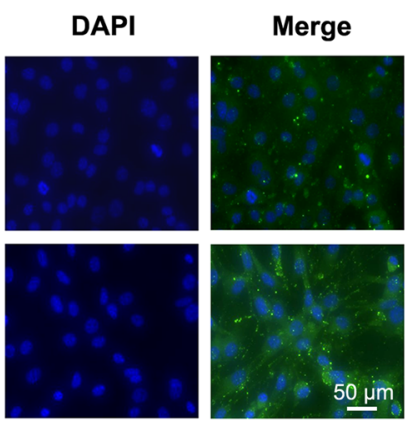

d
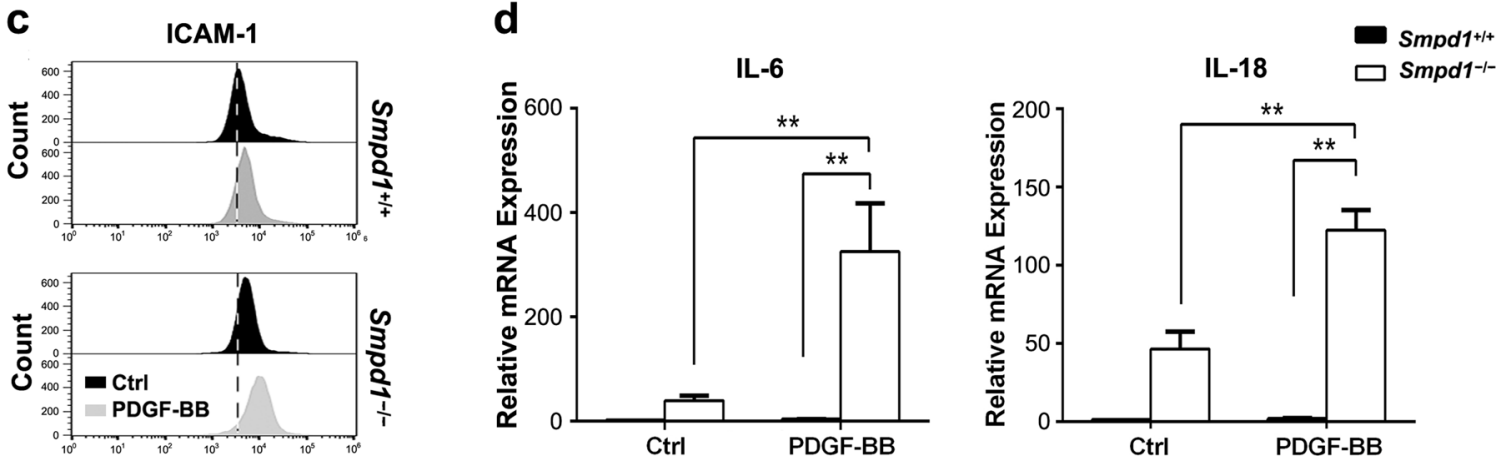

Fig. 4 Smpd1 gene ablation exacerbates PDGF-induced inflammation in SMCs. a Representative images (left) and cell quantification (right) showing the effects of ASM deficiency and PDGF-BB stimulation on monocyte (green) recruitment. SMCs were pretreated with $30 \mathrm{ng} / \mathrm{ml}$ PDGF-BB or equal amount of vehicle for $24 \mathrm{~h}$ and then co-cultured with calcium-AM-labeled monocytes for $30 \mathrm{~min}$. Microscopic observation was conducted with a fluorescence microscope and cell counting was performed by flow cytometry by gating calcium positive cell. Magnification, $\times 200$. ${ }^{*} P<0.05,{ }^{* *} P<$ $0.01(n=4)$. Immunofluorescence $(\mathbf{b})$ and flow cytometry $(\mathbf{c})$ analysis of intercellular adhesion molecule-1 (ICAM- 1 ) expression in $5 \mathrm{mpd} 1^{+/+}$and

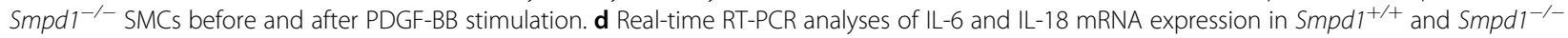
SMCs before and after PDGF-BB stimulation. ${ }^{*} P<0.05,{ }^{* *} P<0.01(n=6)$ 


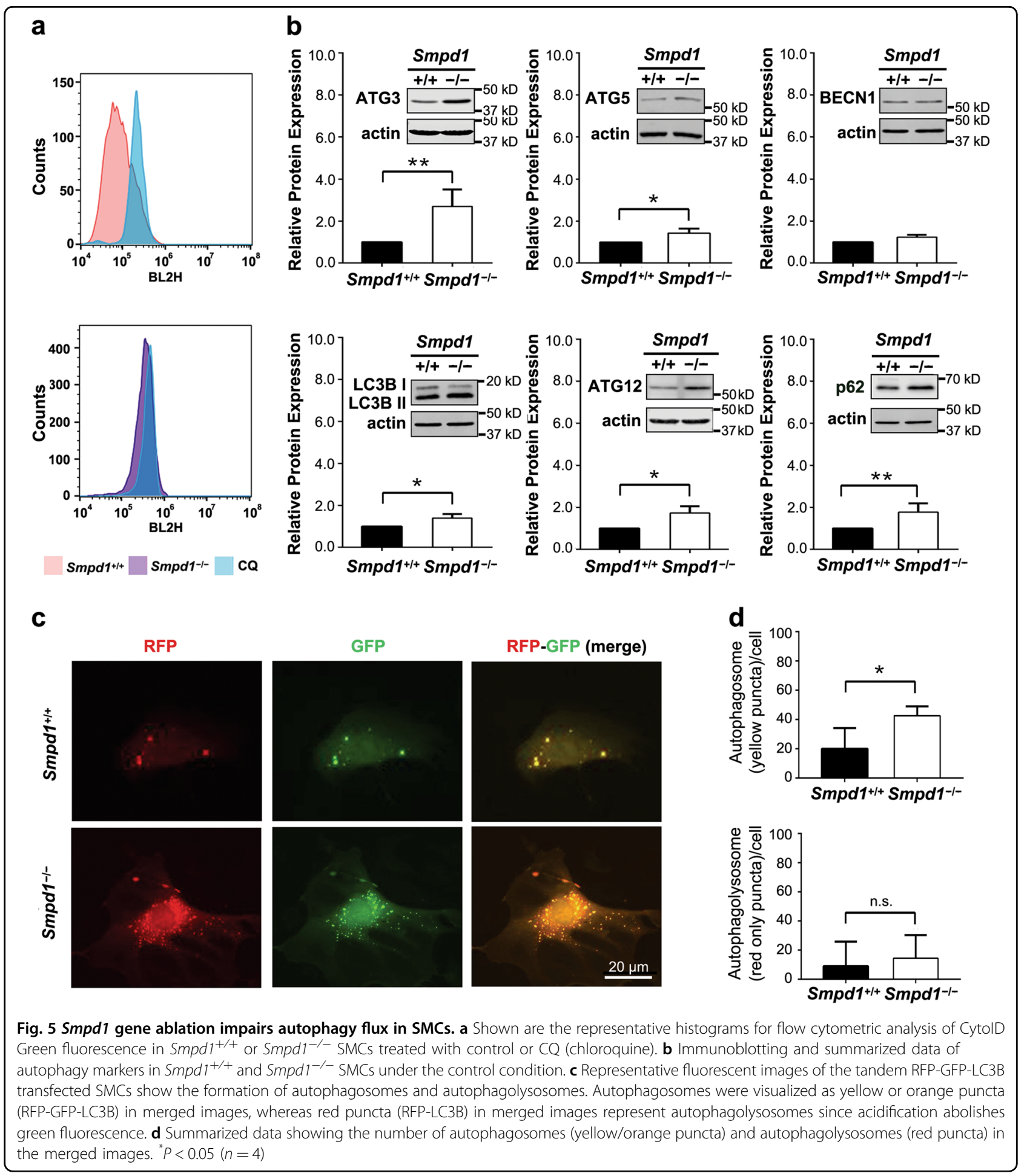

selective autophagy substrate p62/SQSTM1 (Fig. 5b). Thus, these results suggest that accumulation of autophagic vacuoles in Smpd1 $1^{-1-}$ SMCs is related with insufficient autophagic flux but not autophagy induction. Additionally, the autophagosomes and autophagolysosomes were visualized by fluorescence microscopy in
SMCs transfected with tandem RFP-GFP-LC3B plasmids. Accumulation of autophagosomes (yellow $\mathrm{GFP}^{+} \mathrm{RPF}^{+}$ puncta in merged image) was found in Smpd1 $1^{-/}$SMCs, whereas no significant difference in the number of autophagolysosomes (red GFP ${ }^{-}$FP $^{+}$puncta in merged images) was found (Fig. 5c, d). The increased ratio of 
autophagosomes over autophagolysosomes indicates a reduced autophagic flux in Smpd $1^{-1-}$ SMCs. Collectively, these results suggest that SMCs lacking Smpd1 gene exhibit reduced autophagic flux leading to the accumulation of autophagosomes and its substrate p62/SQSTM1.

\section{Inhibition of autophagosome formation by PDGF-BB is essential for myofibroblast-like transition in Smpd $1^{-/-}$ SMCs}

As shown in Fig. 6a, PDGF-BB treatment significantly enhanced p62/SQSTM1 expression in Smpd1 $1^{-/-}$SMCs compared to Smpd1 $1^{+/+}$cells. Moreover, PDGF-BB reduced LC3B-II in Smpd1 $1^{-/}$SMCs but not in Smpd1 $1^{+/+}$SMCs (Fig. 6a). Consistently, PDGF-BB also reduced LC3B in Smpd1 ${ }^{-1-}$ SMCs transfected with GFPLC3 plasmid (Fig. 6b). LC3B-II expression reflects the dynamic balance of autophagosome biogenesis and its turnover via autophagic flux. Thus, these results suggest that in Smpd1 $1^{-/-}$SMCs, impaired autophagic flux leads to $\mathrm{p} 62 / \mathrm{SQSTM} 1$ accumulation, which was further exacerbated by PDGF-BB as it reduces the autophagosome biogenesis. Inhibition of mTOR by rapamycin is commonly used to stimulate autophagy by inducing autophagosome formation in mammalian cells. Interestingly, a

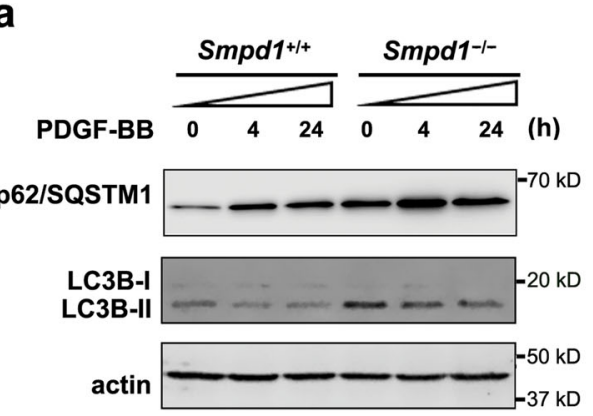

b
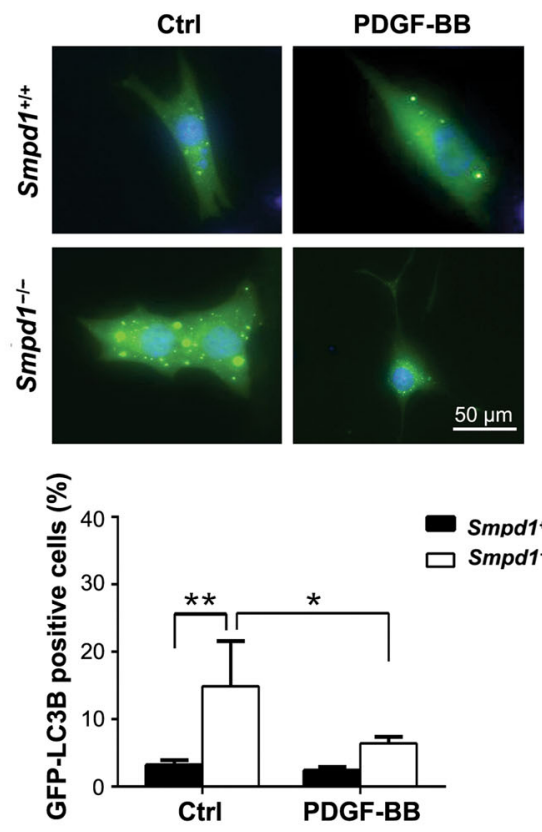
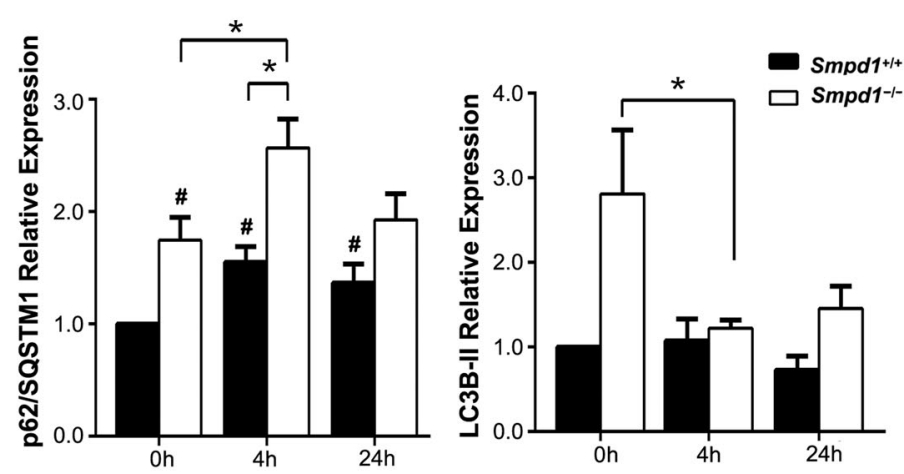

C

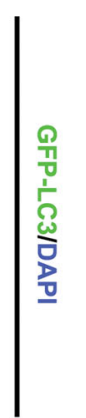

C
PDGF-BB
Rapamycin

Smpd1+/+
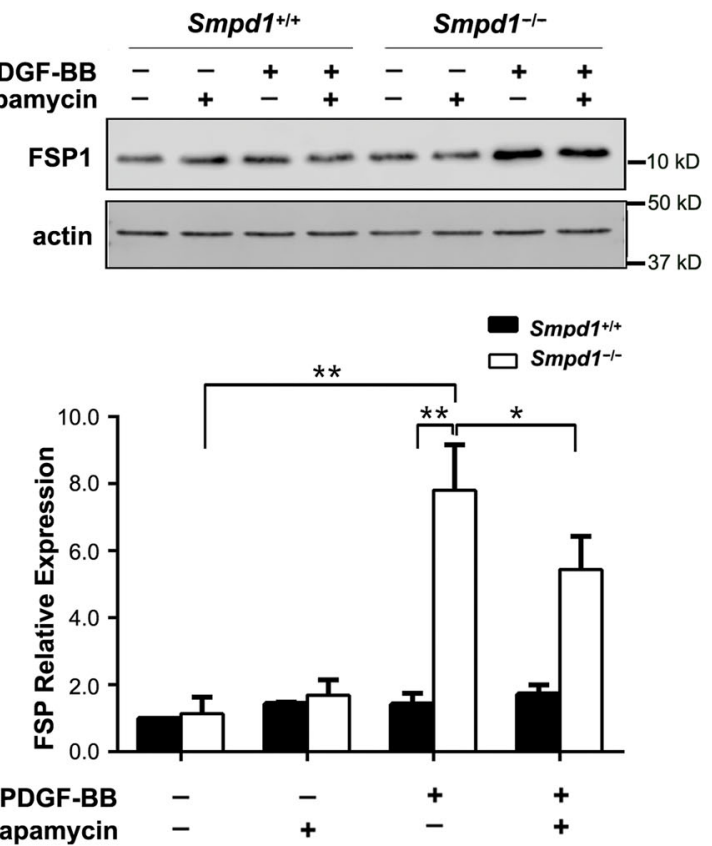

Fig. 6 Autophagy inhibition is required for PDGF-BB-induced myofibroblast-like transition of Smpd $1^{-/-}$SMCs. a Immunoblotting and summarized data of p62/SQSTM1 and LC3B in Smpd $1^{+/+}$or Smpd1 ${ }^{-/-}$SMCs treated with control or PDGF-BB (30 ng/ml) for indicated time. ${ }^{* P}<0.05$, ${ }^{*} P<0.01(n=4)$. $\mathbf{b}$ GFP-LC3B-expressing SMCs were seeded in 8-well Lab-Tek ${ }^{\circledR}$ Chamber Slide, allowed to adapt for $12 \mathrm{~h}$, and maintained in control conditions or exposed to PDGF-BB $(30 \mathrm{ng} / \mathrm{ml})$ for $4 \mathrm{~h}$. Representative images and quantitative data are reported. ${ }^{*} P<0.05$, ${ }^{*} P<0.01(n=6)$. c Immunoblotting and summarized data of FSP-1 in Smpd $1^{+/+}$or Smpd $1^{-/-}$SMCs treated with control or PDGF-BB (30 ng/ml) in the presence or absence of rapamycin $(50 \mathrm{nmol} / \mathrm{l})$ for indicated time. ${ }^{*} P<0.05,{ }^{* *} P<0.01(n=6)$. 
rapamycin partially attenuated PDGF-BB-induced FSP-1 expression in Smpd1 ${ }^{-1-}$ SMCs (Fig. 6c). As a comparison, neither rapamycin nor its combination with PDGF-BB had any effect on FSP-1 in Smpd1 ${ }^{+/+}$SMCs. These results suggested that restoration of autophagy may at least partially prevent PDGF-BB-induced myofibroblast-like transition in Smpd1 $1^{-/-}$SMCs.

\section{Sustained Akt activation is essential for PDGF-BB-induced} myofibroblast-like transition of Smpd $1^{-/}$SMCs

Multiple survival signaling pathways such as Akt and Erk $1 / 2$ are activated by PDGF in SMCs, both of which can activate mTOR signaling that inhibits autophagy induction $^{24}$. As shown in Fig. 7a, a more sustained activation of Akt was induced by PDGF-BB in Smpd1 ${ }^{-/-}$SMCs compared to that in $S m p d 1^{+/+}$SMCs. In contrast, there was no significant difference in Erk1/2 activation between Smpd $1^{+/+}$and $S m p d 1^{-1-}$ cells (data not shown). LY294002, an Akt inhibitor, efficiently suppressed PDGFBB-induced Akt activation in both $S m p d 1^{+/+}$and Smpd1 $1^{-/-}$cells (Fig. 7b). More importantly, in Smpd1 $1^{-/-}$ SMCs, the Akt suppression by LY294002 was accompanied by significant reduction of PDGF-BB-induced p62/ SQSTM1 accumulation and FSP-1 (Fig. 7c). Interestingly, LY294002 markedly attenuated PDGF-BB-induced mRNA level of IL-6 but not IL-18 in Smpd1 $1^{-1-}$ SMCs (Fig. 7d). Further, LY294002 markedly reversed morphological changes by PDGF-BB in Smpd1 $1^{-/}$SMCs as evidenced by the reduction of prolonged cytoplasmic projections and restoration of cell size (Fig. 7e). Altogether, these results raise a possibility that sustained Akt activation inhibits autophagosome formation and thereby contributes to myofibroblast transition.

\section{Akt-p62/SQSTM1 axis contributes to PDGF-induced myofibroblast transition in Smpd $1^{-/-}$SMCs}

As shown in Fig. 8a, b, p62/SQSTM1 gene silencing markedly inhibited PDGF-BB-induced expression of IL-6 but not IL-18 in Smpd1 ${ }^{-/-}$SMCs. p62/SQSTM1 gene silencing almost diminished FSP-1 expression induced by PDGF-BB but had no effect on TGF- $\beta 1$ (Fig. 8c). FSP-1 gene expression was more drastically reduced by $\mathrm{p} 62 /$ SQSTM1 silencing compared to IL-6, which might indicate that FSP-1 gene requires a higher threshold of p62/ SQSTM1 level for its gene induction. These findings suggest that Akt-p62/SQSTM1 pathway contributes to the myofibroblast transition of Smpd1 $1^{-/-}$SMCs upon PDGF-BB stimulation.

\section{Involvement of Nrf2 in PDGF-BB-induced IL-6 production in Smpd $1^{-/-}$SMCs}

Recently studies demonstrated a role of p62/SQSTM1 in linking autophagy and Nrf2 signaling. Accumulated p62/SQSTM1 causes a non-canonical activation of Nrf2 leading to cell proliferation and differentiation ${ }^{25}$. As shown in Fig. 9a, more significant Nrf2 upregulation was observed in Smpd1 $1^{-/}$SMCs treated with PDGF-BB compared to Smpd1 $1^{+/+}$SMCs, especially in the nuclear fraction. Consistently, PDGF-BB induces a more pronounced nuclear translocation of Nrf2 in Smpd1 $1^{-/-}$ SMCs than Smpd1 $1^{+/+}$SMCs (Fig. 9b). Further, Nrf2 gene silencing significantly decreased Nrf2 expression and abolished PDGF-BB induced IL-6 production in Smpd1 $1^{-\prime}$ ${ }^{-}$SMCs (Fig. 9c, d). Collectively, these results demonstrated that Nrf2 plays a role in $S m p d 1^{-/-}$SMCs differentiation.

\section{Discussion}

In the present study, we demonstrated that PDGF-BB induced a myofibroblast-like phenotype in $S m p d 1^{-/-}$ SMCs, which is distinct from PDGF-BB-induced "canonical" synthetic phenotype in Smpd1 $1^{+/+}$SMCs. This unique myofibroblast-like "non-canonical" synthetic phenotype in Smpd1 $1^{-/-}$SMCs is characterized by: (1) morphological changes with extended pseudopodia elongation and cell shrinkage; (2) elevated expression of fibroblast marker FSP-1; (3) enhanced extracellular deposition of collagens; (4) massive production of inflammatory cytokines. Our findings further demonstrated that ASM participates in PDGF-BB-induced SMC myofibrogenic switching by modulating Akt-autophagyp62/SQSTM1 axis.

PDGF-induced SMC proliferation and migration are essential processes in vascular disorders such as atherosclerosis $^{1,26}$. Our data showed that PDGF-BB induced similar changes in cell cycle, cyclin D1 expression, or cell numbers in between $S m p d 1^{-/-}$and $S m p d 1^{+/+}$cells. Further, ASM deficiency had no effect on PDGF-BBinduced outgrowth of sprouts from aortic explants. Our data, thus, reveal that PDGF-BB-treated Smpd1 $1^{-1-}$ SMCs remain proliferative and migratory, which resembles "canonical" synthetic phenotype. PDGF-BB induced obvious morphological changes in Smpd1 $1^{-1-}$ SMCs with extended pseudopodia elongation and cell shrinkage, whereas Smpd1 $1^{+/+}$SMCs have a spindle-shaped appearance. We also found that PDGF-BB-treated Smpd1 $1^{-/-}$ SMCs rapidly reduced their cell size as reflected by decreased cell impedance, particularly in the early stage of PDGF-BB stimulation. Such decrease in cell size raises a possibility that dedifferentiated Smpd1 $1^{-1-}$ SMCs convert readily to a myofibroblastic-like phenotype that is different from "canonical" synthetic phenotype observed in Smpd $1^{+/+}$SMCs.

So far, there is no specific marker to discriminate myofibroblasts from fibroblasts or SMCs. Myofibroblasts have a phenotype intermediate between fibroblasts and SMCs. The myofibroblast-like phenotype may possess the differentiation markers of fibroblasts and SMCs. It is 


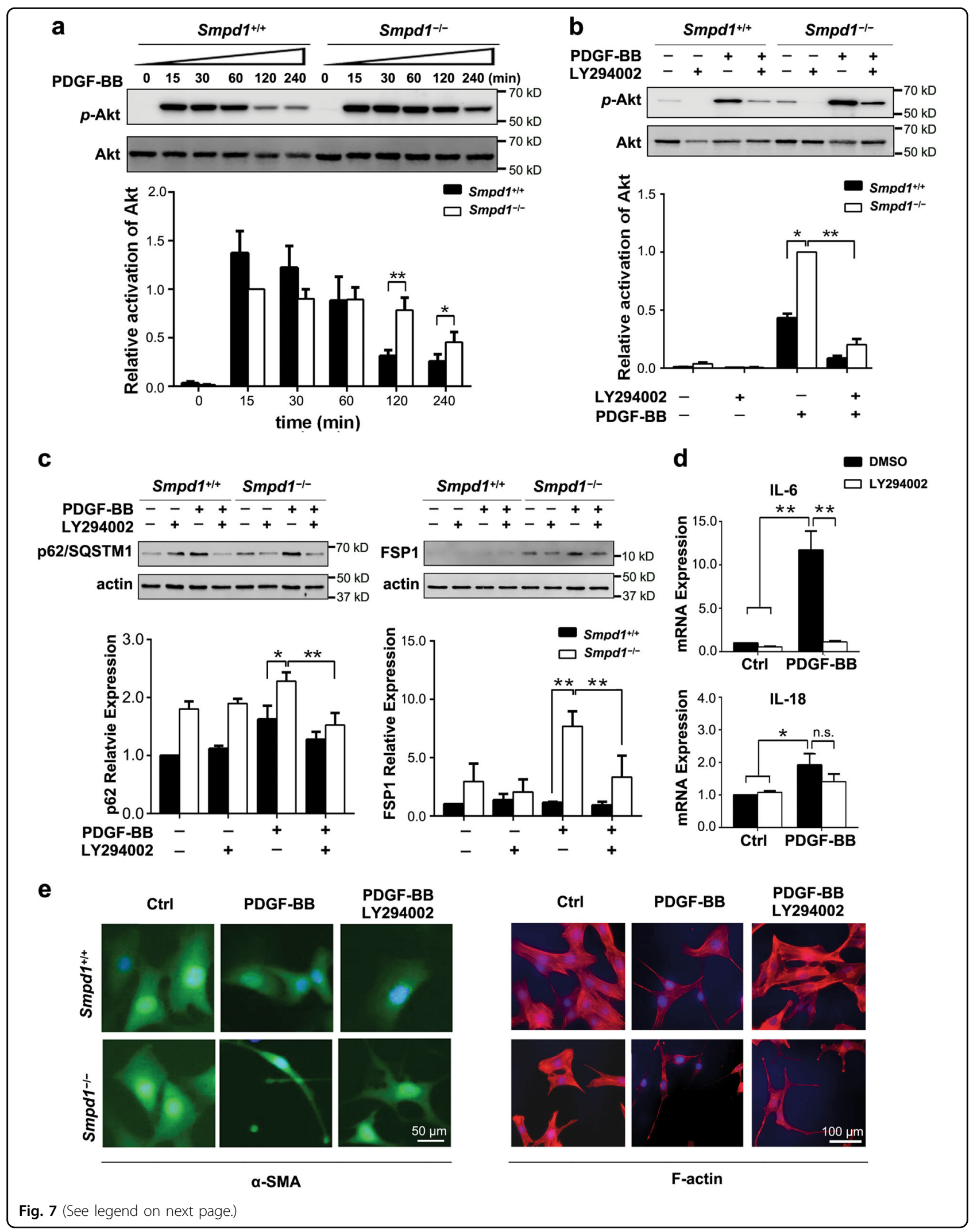


(see figure on previous page)

Fig. 7 Sustained Akt activation is required for in PDGF-BB induced myofibroblast transition of Smpd $1^{-1-}$ SMCs. a Representative images and quantitative data show the effects of PDGF-BB $(30 \mathrm{ng} / \mathrm{ml})$ on the phosphorylation of Akt and Erk-1/2 in in Smpd1 ${ }^{+/+}$or Smpd1 ${ }^{-/-} \mathrm{SMCs} .{ }^{*} P<0.05$, ${ }^{*} P<0.01(n=4)$. b, c Representative images and quantitative data show the effect of Akt inhibitor LY294002 (50umol/l) on PDGF-BB-induced changes in Akt phosphorylation, p62/SQSTM1, and FSP-1 in Smpd1 $1^{+/+}$or Smpd1 ${ }^{-1-}$ SMCs. SMCs were treated with or without PDGF-BB (30 ng/ml) for $2 \mathrm{~h}$ for Akt phosphorylation or for $24 \mathrm{~h}$ for analysis of p62/SQSTM1 and FSP-1. ${ }^{*} P<0.05,{ }^{* *} P<0.01(n=4)$. d Real-time RT-PCR analyses of IL-6 and

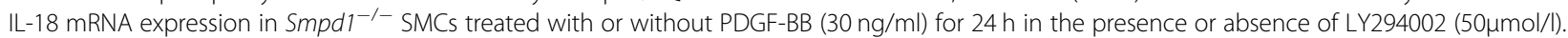
${ }^{*} P<0.05,{ }^{* *} P<0.01(n=6)$. e Representative immunofluorescence images of Smpd $1^{+/+}$or Smpd $1^{-1}$ SMCs treated with or without PDGF-BB (30 ng/ $\mathrm{ml}$ ) in the presence or absence of LY294002 $(50 \mu \mathrm{mol} / \mathrm{l})$ for $24 \mathrm{~h}$. The experiment was repeated at least three times
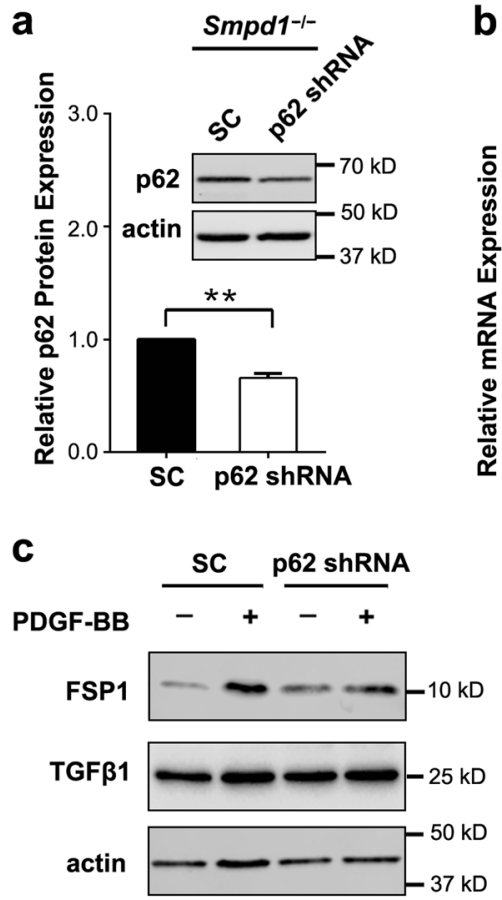

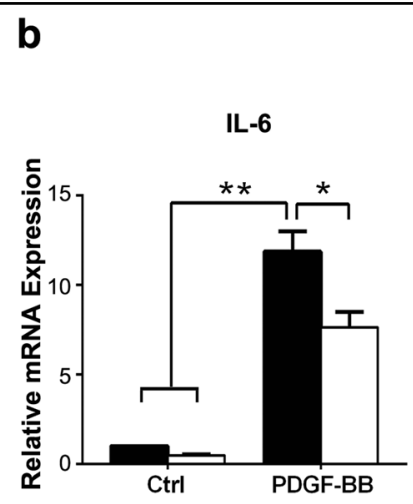

FSP1

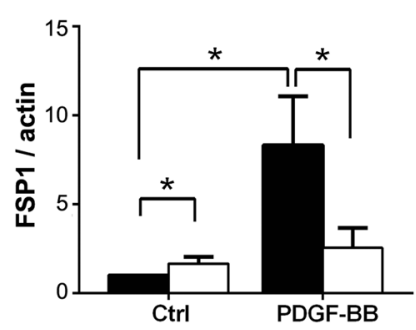

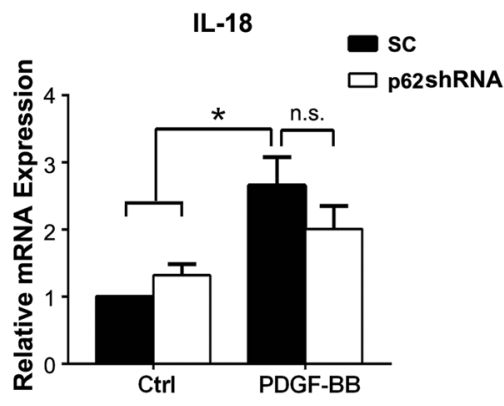

TGF $\beta 1$

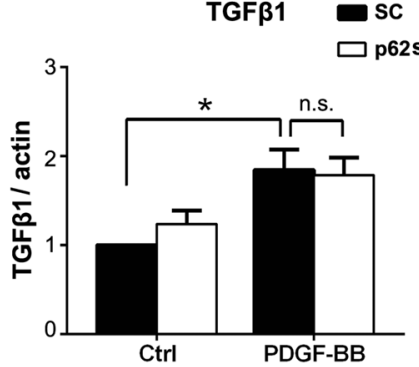

Fig. 8 Akt-p62/SQSTM1 axis contributes to PDGF-BB induced myofibroblast-like phenotype transition of Smpd1 ${ }^{-/-}$SMCs. Smpd1 $^{-/-}$SMCs were infected with p62/SQSTM1 shRNA or scramble shRNA lentiviral particles and then treated with PDGF-BB (30 ng/ml) for $24 \mathrm{~h}$. a Representative immunoblotting and summarized data show the protein expression of p62/SQSTM1 in Smpd1 ${ }^{-/-}$SMCs infected with p62/SQSTM1 shRNA or scramble shRNA (SC) lentiviral particles. ${ }^{*} P<0.05,{ }^{*} P<0.01(n=3)$. b Real-time RT-PCR analyses of IL-6 and IL-18 mRNA expression in PDGF-BB or vehicle treated Smpd1 ${ }^{-1-}$ SMCs with p62/SQSTM1 silencing or not. ${ }^{*} P<0.05,{ }^{* *} P<0.01$ ( $n=6$ ). c Representative immunoblotting and summarized data show the effects of p62/SQSTM1 shRNA on PDGF-BB-induced protein expression of FSP-1 and TGF- $\beta 1$ in Smpd1 ${ }^{-1-}$ SMCs

necessary to compare multiple SMC differentiation marker and fibroblast marker between the morphologically different phenotypes. Several SMC markers ( $\alpha$-SMA, calponin, SM22) are differently expressed in myofibroblast-like Smpd1 $1^{-/-}$SMCs compared to "canonical" synthetic Smpd1 $1^{+/+}$SMCs. Among them, the expression level of $\alpha$-SMA is lower in Smpd1 $1^{-/}$SMCs than Smpd $1^{+/+}$SMCs. Although $\alpha-S M A$ is a classic SMC marker and not expressed in endothelial cells or fibroblasts, it is also a commonly used marker to identify myofibroblast from fibroblast in fibrotic diseases ${ }^{27}$. The SMC dedifferentiation is associated with decreased expression of $\alpha-\mathrm{SMA}^{28}$. Therefore, the lower expression of $\alpha$-SMA in myofibroblast-like Smpd1 $1^{-/-}$SMCs suggest a more dedifferentiated state in these cells than "canonical" synthetic Smpd1 $1^{+/+}$SMCs. Interestingly, myofibroblastic-like Smpd1 $1^{-/}$SMCs have increased expression of contractile markers calponin and SM22. Calponin is a calmodulin, F-actin and tropomyosin binding protein that is involved in smooth muscle contraction $^{29}$. SM22 is a transformation and shape-change sensitive actin cross-linking/gelling protein ${ }^{30}$. As calponin and SM22 are contraction and shape-related proteins, the increases in calponin and SM22 may be associated with the alteration in contractility and/or morphological changes during myofibroblast-like transition. Further, myofibroblast-like Smpd $1^{-/-}$SMCs induced by PDGF-BB are highly expressed in FSP-1, have enhanced collagen deposition and TGF- $\beta 1$ secretion, and demonstrate increased adhesion to monocytes and massive production 


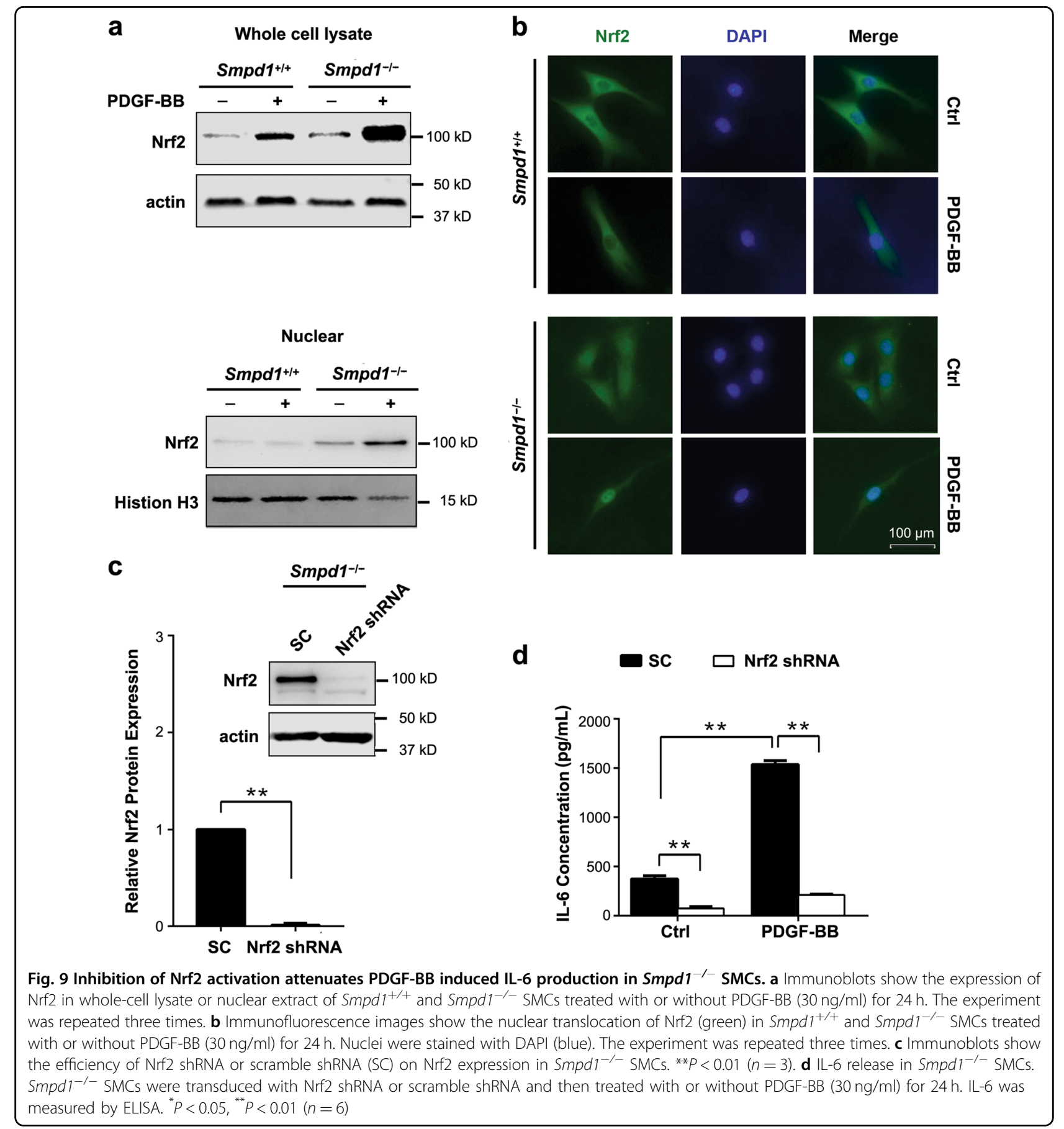

inflammatory cytokines (IL-6 and IL-18), all of which are commonly found in activated myofibroblasts ${ }^{31}$. To our knowledge, our findings provide the first evidence that PDGF-BB promoted a myofibroblastic transition in Smpd $1^{-/-}$SMCs.

Recent studies demonstrated that moderately enhanced autophagy in the vasculature protects against progressive atherosclerosis or restenosis ${ }^{32}$. Autophagy blunts proliferation in SMCs under various atherogenic stimuli such as thrombin and advanced glycation end products ${ }^{33,34}$.
Enhanced autophagy by either statins or rapamycin helps maintain vascular smooth muscle in contractile phenotype and inhibits their proliferation ${ }^{18,32}$. ASM-deficient SMCs exhibits reduced autophagic flux that contributes to enhanced proliferation by 7 -ketocholesterol ${ }^{17}$. These previous studies suggest that ASM-mediated autophagy plays a protective role in SMCs by maintaining contractile phenotype. PDGF-BB increases autophagosome induction and inhibition of autophagy prevents PDGF-BB-induced synthetic phenotype transition in rat aortic SMCs, which 
suggest that autophagy is critical for attaining the synthetic phenotype ${ }^{35,36}$. However, ASM deficiency had no effect on PDGF-BB-induced proliferation in SMCs despite these cells had reduced autophagic flux. Moreover, PDGF-BB caused an inhibition of autophagosome biogenesis in Smpd1 $1^{-/}$SMCs, which was concomitant with augmented p62/SQSTM1 expression. Interestingly, reactivation of autophagy by rapamycin, an inhibitor of mTOR, markedly attenuated PDGF-BB-induced FSP-1 expression in Smpd1 $1^{-1-}$ SMCs. Therefore, our findings support the view that PDGF-BB-induced autophagosome inhibition and defective autophagic flux act synergistically to induce or promote myofibroblastic transition in Smpd $1^{-/-}$SMCs.

Moreover, this study reveals that sustained activation of Akt signaling by PDGF-BB leads to the inhibition of autophagosome biogenesis, which contributes to enhanced p62/SQSTM1 accumulation in Smpd1 $1^{-/-}$ SMCs. PDGF-BB binds with PDGF receptors leading to activation of pathways such as Akt and mitogen-activated protein kinase Erk1/2 cascades $^{37}$. Both Akt and Erk1/2 pathways regulate autophagy signaling as well as proliferation of mammalian cells ${ }^{38,39}$. In Smpd $1^{-/-}$SMCs, PDGF-BB induced a sustained activation of Akt but not Erk1/2 signaling. Ceramide, the hydrolysis product of sphingomyelin by ASM, activates phosphatase $2 \mathrm{~A}$ (PP2A), which dephosphorylates and inhibits $\mathrm{Akt}^{40}$. The sustained Akt activation in Smpd1 $1^{-/-}$SMCs could be due to the absence of ceramide-PP2A axis. Targeting Akt signaling by LY294002 attenuated PDGF-BB-induced expression of p62/SQSTM1 and FSP-1, reduction of cell size, and production of IL-6 in Smpd1 $1^{-/}$SMCs. Altogether, our findings indicate a causative role of sustained Akt activation in the inhibition of autophagosome biogenesis, which importantly contributes to myofibroblastic transition in Smpd1 $1^{-1-}$ SMCs.

The present study further investigated the molecular mechanisms linking diminished autophagy signaling and myofibroblastic transition. p62/SQSTM1 participates in epithelial-to-myofibroblast transition (EMT) that podocytes lose epithelial features and acquire myofibroblast features such as increased expression of FSP-1 and $\alpha$ $\mathrm{SMA}^{41}$. Similarly, the present study for the first time demonstrated a critical role of the autophagy-p62/ SQSTM1 axis in several key characteristics of myofibroblast-like phenotype including FSP-1 and IL-6 expression. However, PDGF-BB-induced TGF $\beta 1$ expression and IL-18 production are independent of p62/ SQSTM1 signaling. Therefore, our data suggest that the myofibroblastic transition of Smpd1 $1^{-/-}$SMCs is through both p62SQSTM1-dependent and -independent pathways. p62/SQSTM1 may link to several signaling pathways such as cyclin-dependent kinase 1 (CDK1)-mediated p62 phosphorylation, Nrf2-mediated redox signaling, and
NF-kB-dependent transcriptional regulation ${ }^{42,43}$. We demonstrated that PDGF-BB caused abnormal Nrf2 activation in Smpd1 $1^{-1-}$ SMCs, which contributes to IL-6 production. Therefore, these results suggest that Nrf2 may be a downstream target of p62/SQSTM1 to induce IL-6. Future investigation is needed to decipher the precise mechanism by which autophagy-p62/SQSTM1 axis or $\mathrm{p} 62 / \mathrm{SQSTM} 1$-independent pathway triggers the myofibroblastic transition.

In summary, our work highlights the importance of ASM-autophagy cascade in myofibroblastic transition. As depicted in Fig. 10, in our system, ASM modulates both autophagosome biogenesis and its lysosome-mediated autophagic flux pathway. Ceramide produced by ASM activity activates PP2A and limits PDGF-BB-induced Akt and $\mathrm{mTOR}$ activation. ASM deficiency leads to reduced ceramide production, deactivation of $\mathrm{PP} 2 \mathrm{~A}$, and consequent long-term activation of Akt-mTOR signaling. Such long-term Akt-mTOR activation suppresses autophagosome biogenesis. In another aspect, ASM activity is needed for autophagic flux. ASM deficiency reduces autophagic flux leading to delayed turnover of autophagosomes. Therefore, in situation of ASM deficiency, reduced autophagosome biogenesis and turnover synergistically cause diminished autophagy signaling leading to exacerbated accumulation of p62/SQSTM1 that may trigger its downstream activation of myofibroblastic reprogramming such as expression of FSP-1 and IL-6 genes. These results provide novel insights into a new therapeutical intervention for preventing SMCmyofibroblast transition in vascular diseases such as atherosclerosis.

\section{Materials and methods \\ Mice}

ASM-deficient (Smpd1 ${ }^{-1}$; Smpd1 is the gene symbol for ASM gene sphingomyelin phosphodiesterase 1) and wild-

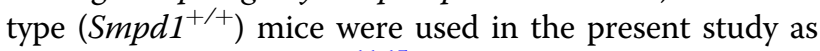
we described previously ${ }^{44,45}$. All experimental protocols were reviewed and approved by the Animal Care Committee of the University of Houston. All animals were provided with standard rodent chow and water ad libitum in a temperature-controlled room.

\section{Antibodies and reagents}

Mouse recombinant PDGF-BB (SRP3229), Direct Red 80 (365548), picric acid (197378), Fast green FCF (F7252), and anti- $\alpha$-smooth muscle actin mouse mAb (C6189) were purchased from Sigma-Aldrich (St. Louis, MO, USA). Antibodies against mouse Collagen I (ab21286), Vimentin (ab92547), $\alpha$-smooth muscle actin (ab5694), ICAM1(ab119871), SM22 alpha (ab14106), P62/SQSTM1 (ab109012) were from Abcam (Cambridge, UK). Antimouse Atg3 (\#3415), Atg5 (\#12994), Beclin-1 (\#3495), 


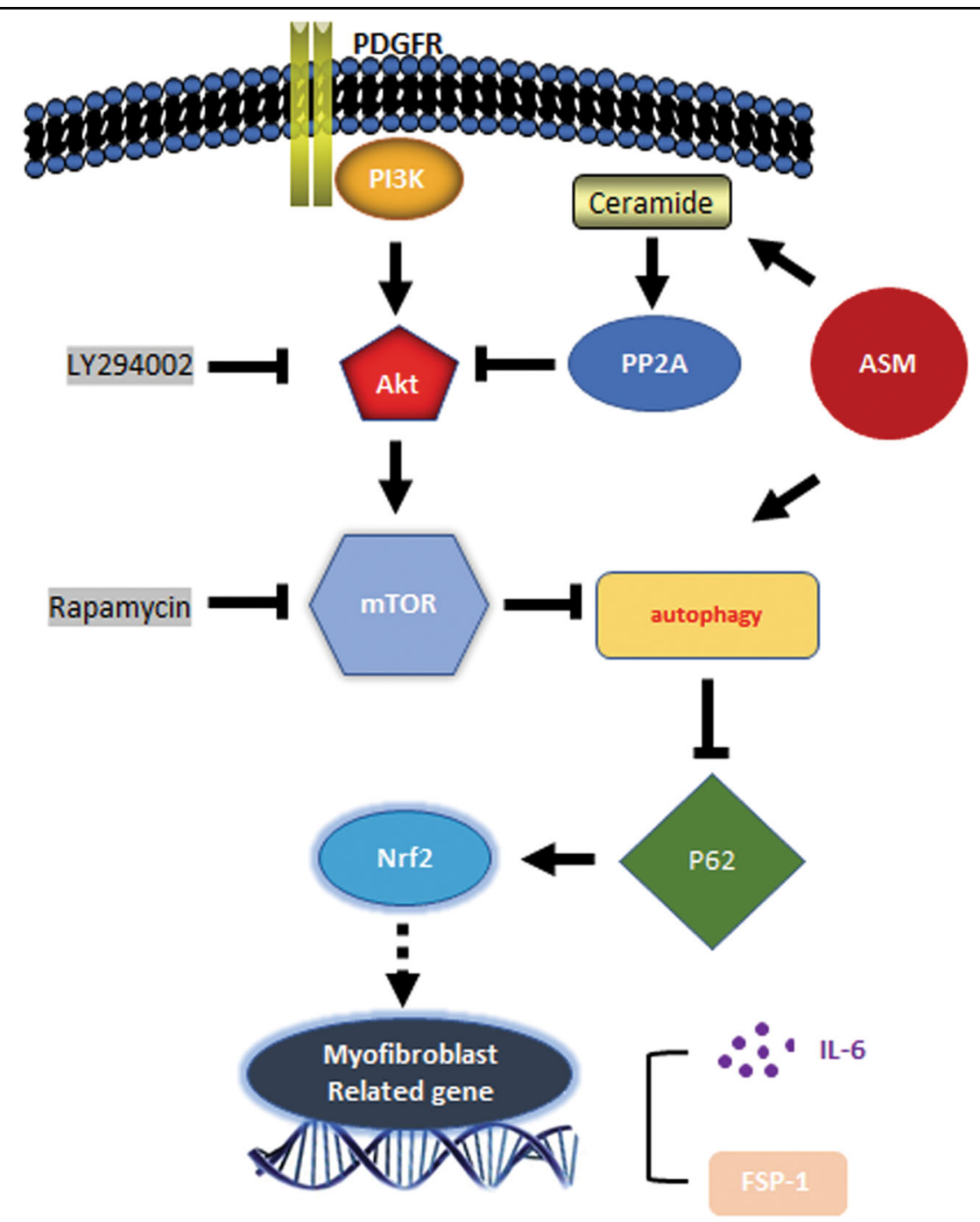

Fig. 10 Proposed mechanism by which ASM deficiency accelerates myofibroblast-like phenotype transition in Smpd $1^{-/-}$upon PDGF-BB stimulation (see discussion for detail description)

LC3A/B (\#12741), Atg12 (\#4180), phospho-Akt (\#4060), Akt (\#9272), phospho-p44/42 MAPK (Erk1/2) (\#4370), p44/42 MAPK (Erk1/2) (\#4695), phospho-AMPK $\alpha$ (Thr172) (\#2535), AMPKa (\#2532), phospho-mTOR (Ser2448) (\#5536), mTOR (\#2983), $\beta$-actin (\#8457), TGF- $\beta$, and Fibroblast-specific protein 1 (13018) were from Cell Signaling Technology (Danvers, MA, USA). Antibody specific to Cyclin D1 (554181) was obtained from BD Pharmingen. IRDye ${ }^{\circledR} 800 \mathrm{CW}$ Goat anti-Rabbit and IRDye ${ }^{\circledR} 680 \mathrm{CW}$ goat anti-mouse secondary antibodies were from LI-COR (Lincoln, NE, USA). Alexa Fluor 488 and Alexa Fluor ${ }^{\circledast} 555$ conjugated secondary antibodies, ActinRed $^{\mathrm{TM}} 555$ ReadyProbes $^{\circledR}$ Reagent (R37112), Premo ${ }^{\mathrm{TM}}$ Autophagy Tandem Sensor GFP-LC3B Kit (P36239), FxCycle ${ }^{\mathrm{TM}}$ PI/RNase Staining Solution (F10797) and Fast-red were purchased from Thermo Fisher Scientific (Waltham, MA, USA). CYTO-ID ${ }^{\circledR}$
Autophagy Detection Kit 2.0 was obtained from Enzo Life Sciences (New York, NY, USA). Sqstm1-mouse shRNA lentiviral particles (TL515047V) were prepared by Origene (Rockville, MD, USA).

\section{Primary culture of SMCs}

Mouse coronary arterial SMCs were isolated as previously described ${ }^{46}$. Six-week-old male C57BL/6J ASMdeficient $\left(\operatorname{Smpd1} 1^{-/-}\right)$mice and their wild-type littermates $\left(\operatorname{Smpd} 1^{+/+}\right)$were used in the present study. In brief, mice were deeply anesthetized with intraperitoneal injection of pentobarbital sodium $(25 \mathrm{mg} / \mathrm{kg})$. The heart was excised with an intact aortic arch and immersed in a petri dish filled with ice-cold Krebs-Henseleit solution. A 25-gauge needle filled with Hanks' buffered saline solution was inserted into the aortic lumen opening while the whole heart remained in the ice-cold buffer solution. The 
opening of the needle was inserted deep into the heart close to the aortic valve. The needle was tied in place with the needle tip as close to the base of the heart as possible. The infusion pump was started with a 20-ml syringe containing warm HBSS through an intravenous extension set at a rate of $0.1 \mathrm{ml} / \mathrm{min}$ for $15 \mathrm{~min}$. HBSS was replaced with warm enzyme solution $(1 \mathrm{mg} / \mathrm{ml}$ collagenase type I, $0.5 \mathrm{mg} / \mathrm{ml}$ soybean trypsin inhibitor, $3 \%$ bovine serum albumin, and $2 \%$ antibiotic), which was flushed through the heart at a rate of $0.1 \mathrm{ml} / \mathrm{min}$. Perfusion fluid was collected at 30,60, and 90-min intervals. At $90 \mathrm{~min}$, the heart was cut with scissors, and the apex was opened to flush out the cells that collected inside the ventricle. The fluid was centrifuged at $1000 \mathrm{rpm}$ for $10 \mathrm{~min}$, the cell-rich pellets were mixed with the media described below, and the cells were plated on $2 \%$ gelatin-coated six-well plates and incubated in $5 \% \mathrm{CO}_{2}$ at $37^{\circ} \mathrm{C}$. Advanced Dulbecco's modified Eagle's medium (DMEM) with $10 \%$ fetal bovine serum, $10 \%$ mouse serum, and $2 \%$ antibiotics were used for isolated SMCs. The identification of SMCs was based on positive staining by anti- $\alpha$-actin antibody and the SMC morphology. The medium was replaced 3 days after cell isolation and then once or twice each week until the cells grew to confluence. All studies were performed with cells of passage of 3-6.

\section{Electric cell-substrate impedance sensing (ECIS) assay}

ECIS can detect and quantify morphology changes in the sub-nanometer to micrometer range. The impedance is related to the number of cells covering the electrode, the morphology of the cells, and the nature of the cell attachment. The changes in cell function may alter its morphology by decreasing its size and thereby decrease the impedance. The ECIS $^{\circledR} \mathrm{Z} \theta$ (theta) (Applied BioPhysics, USA) was used to monitor cell behavior according to the manufacturer's instructions. Briefly, before starting ECIS measurements, $200 \mu \mathrm{l}$ of complete medium was placed in each well and allowed to equilibrate in the incubator for $30 \mathrm{~min}$. Then $2 \times 10^{5} \mathrm{Smpd1^{+/+ }}$ and Smpd1 $1^{-1-}$ SMCs were separately seeded in 8W10E + plates containing gold film surface electrodes in DMEM supplemented with $10 \%$ fetal bovine serum (FBS). When cells attached and spread to the electrodes of ECIS Arrays, they act as insulators increasing the impedance. As cells grow and cover the electrodes, the current is impeded in a manner related to the number of cells covering the electrode, the morphology of the cells and the nature of the cell attachment. When cells are stimulated to change their function, the accompanying changes in cell morphology alter the impedance. The cells were monitored noninvasively under current pulses $40 \mathrm{kHz}$ with $600 \mathrm{~s}$ interval within $72 \mathrm{~h}$. The data generated is impedance versus time.

\section{Immunoblotting}

Cells were lysed in Laemmli sample buffer (Bio-Rad, 161-0737) containing $\beta$-mercaptoethanol (SigmaAldrich, M3148) and boiled for $10 \mathrm{~min}$ at $95^{\circ} \mathrm{C} .30 \mu \mathrm{g}$ of total proteins were separated by $8-12 \%$ sodium dodecyl sulfate-polyacrylamide gel electrophoresis. The proteins of these samples were then electrophoretically transferred at $100 \mathrm{~V}$ for $1 \mathrm{~h}$ onto a PVDF membrane (Bio-Rad, USA). The membrane was blocked with $5 \%$ non-fat milk in Trisbuffered saline-Tween 20. After washing, the membrane was probed with primary antibody as indicated according to the manufacturer's instructions. After washing, the membranes were then incubated with IRDye donkey anti-mouse or anti-rabbit fluorescence-conjugated secondary antibodies and bands were visualized and analyzed by LI-COR ${ }^{\circledR}$ Odyssey Fc System.

\section{Cell cycle analysis}

SMCs were plated into 6 well plates at a density of $1 \times$ $10^{6} \mathrm{cells} / \mathrm{ml}$ and incubated for $2 \mathrm{~h}$ treatment with $2 \% \mathrm{FBS}$. Then the medium was replaced with fresh medium containing PDGF-BB $(30 \mathrm{ng} / \mathrm{ml})$ and incubated for $24 \mathrm{~h}$ at 37 ${ }^{\circ} \mathrm{C}$. The cells were harvested washed with ice-cold PBS for three times and then fixed with $70 \%$ ethanol for $30 \mathrm{~min}$ at $4{ }^{\circ} \mathrm{C}$. Subsequently, FxCycle ${ }^{\mathrm{rm}}$ PI/RNase Staining Solution (molecular probes, Life Technologies) was added to the fixed cells. In total, $1 \times 10^{4}$ cells were analyzed by flow cytometry (Attune NxT acoustic focusing cytometer, Life Technologies, USA). All experiments were repeated three times and data analysis was recorded using AttuneR NxT software.

\section{Collagen staining and quantification}

Total collagen amount was assayed using Sirius red staining as previously described ${ }^{47}$. Briefly, the Picro-Sirius red stain solution $(0.1 \%$ direct red 80 plus $0.1 \%$ fast green FCF in saturated aqueous picric acid) was freshly prepared before use. For in vitro culture cell layers, $5 \times 10^{5}$ SMCs were cultured in 24-well plate and then treated with PDGF-BB $(30 \mathrm{ng} / \mathrm{ml})$ or vehicle alone for $24 \mathrm{~h}$. Then removed the culture medium and washed the cells with PBS for three times. The cell layers were completely immersed and fixed with Kahle fixative and incubated for $10 \mathrm{~min}$ at room temperature. After being washed with PBS for three times, cells were incubated with dye solution for $30 \mathrm{~min}$ at room temperature. Carefully removed the dye solution and rinsed the stained cell layers with 0.5 $\mathrm{ml}$ distilled water repeatedly until the fluid is colorless. For paraffin-embedded tissue sections, deparaffinized and hydrated tissue sections were covered with adequate Picro-Sirius red solution and incubated for 60 minutes at room temperature. Then rinsed slide quickly in 10 dips of 
distilled water and quickly dehydrated and mounted in xylene. To calculate the amount of collagen, the following formulas were used: Collagen $(\mu \mathrm{g} / \mathrm{section})=\left[\mathrm{OD}_{540}\right.$ value $-\left(\mathrm{OD}_{605}\right.$ value $\left.\left.\times 0.291\right)\right] / 0.0378$; Non-collagenous proteins $(\mu \mathrm{g} /$ section $)=\mathrm{OD}_{605}$ value $/ 0.00204$.

\section{Monocyte retention and quantification}

Mouse macrophage J774 cells were preincubated in DMEM containing $2 \% \mathrm{FBS}$ and $5 \mu \mathrm{mol} / \mathrm{l}$ fluorescent calcium-AM (C3100MP, Thermo Fisher Scientific, USA) at $37^{\circ} \mathrm{C}$ for $30 \mathrm{~min}$. Fluorescently labeled cells were washed twice to remove unincorporated dye and were then resuspended in DMEM containing 2\% FBS. Loaded monocytic cells $\left(1 \times 10^{5}\right)$ were added to each well of SMCs and incubated at $37^{\circ} \mathrm{C}$. After $30 \mathrm{~min}$, unbound monocytes were withdrawn and SMC layers with attached monocytes were gently washed twice with DMEM. Fluorescence imaging (excitation $485 \mathrm{~nm}$, emission $535 \mathrm{~nm}$ ) was performed using Olympus IX73 imaging system. For cell quantification, the number of recruited J774.2 cells were assessed by flow cytometry to quantify green calcium-AM positive cells.

\section{Quantitative real-time PCR}

Total RNA was isolated using the Aurum Total RNA Mini Kits (Bio-Rad, Cat. \#732-6820, USA) according to the manufacturer's instructions. cDNA was generated from the RNA using iScript Reverse Transcription Supermix for RT-qPCR (Bio-Rad, Cat. \#1708841, USA). Real-Time PCR was performed using the iTaq Universal SYBR Green Supermix (Bio-Rad, Cat. \#1725121, USA) on the Bio-Rad CFX Connect Real-Time System using the following primers: P62/SQSTM1 forward primer: 5'AGGGAACACAGCAAGCT-3'; P62/SQSTM1 reverse primer: 5'-GCCAAAGTGTCCATGTTTCA-3'; GAPDH forward primer: 5' - CCAGAACATCATCCCTGCAT-3'; GAPDH reverse primer: 5'- CAGTGAGCTTCCCGTTCA-3'. Primers for IL-6 and IL-18 were from Bio-Rad PrimePCR ${ }^{\mathrm{mw}} \mathrm{SYBR}^{\circledR}$ Green Assay. The cycle threshold values were converted to relative gene expression levels using the $2^{-\Delta \Delta \mathrm{Ct}}$ method. The data were normalized to that of internal control GAPDH.

\section{Flow cytometric analysis of autophagosomes (APs)}

Autophagic vacuoles, including autophagosomes (APs) and autophagolysosomes (APLs) in cells, were detected using a CytoID Autophagy Detection Kit (Enzo, USA) following the manufacturer's instruction. The CytoID fluorescent reagents specifically detect the autophagic vacuoles formed during autophagy. Briefly, cells were trypsinized, spun down, and washed twice in phenol redfree DMEM with $2 \%$ FBS. The cells were resuspended in $0.5 \mathrm{ml}$ of freshly diluted CytoID reagents and incubated at $37^{\circ} \mathrm{C}$ for $30 \mathrm{~min}$. The CytoID fluorescence of cells was immediately analyzed by flow cytometry using a flow cytometer (Attune NxT acoustic focusing cytometer, Life Technologies, USA). The percentage of cells with CytoID staining was used to represent the dynamic balance between AP formation and its degradation via autophagic flux. By combining with an inhibitor of lysosomal degradation (chloroquine, $30 \mu \mathrm{M}$ ) that inhibits autophagic flux, the CytoID assay can be used to determine whether the autophagic vacuole accumulation is due to decrease in autophagic flux.

\section{Lentiviral transduction}

Both control and the mouse sqstm 1 shRNA lentil particles were purchased from OriGene (TL515047V). Nfe2l2 shRNA plasmid (TL515053) was also obtained from OriGene. Nrf2 shRNA lenti particles were generated according to the manufacturer's instructions. Smpd $1^{-/-}$ SMCs were infected with the lentiviral particle in the presence of polybrene (final concentration was $8 \mu \mathrm{g} / \mathrm{ml}$ ). After $48 \mathrm{~h}$ transduction, puromycin $(1 \mu \mathrm{g} / \mathrm{ml})$ was added to the media for selection. Surviving cells were allowed to proliferate for another $24 \mathrm{~h}$ and were used for downstream analyses. The effect of gene silencing was then analyzed by assessing target mRNA and protein level.

\section{Immunofluorescence staining}

For culture cells, approximately $1 \times 10^{4}$ mouse SMCs were added to the wells of 24-well culture plate containing gelatin-coated coverslips. After being treated with PDGF$\mathrm{BB}(30 \mathrm{ng} / \mathrm{ml})$ or the equivalent volume of the vehicle for $24 \mathrm{~h}$, cells were washed with PBS and then fixed with $4 \%$ paraformaldehyde for 20 minutes at room temperature. After twice washes with PBS, $0.3 \%$ Triton X-100 in PBS was added for $15 \mathrm{~min}$ for cell permeabilization. Nonspecific sites were blocked with PBS with 1\% BSA and 1\% normal donkey serum at room temperature for $1 \mathrm{~h}$. For paraffin-embedded tissue sections, peroxidase treatment in methanol with $0.5 \%$ hydrogen peroxide was followed by heat-assisted antigen retrieval in $0.01 \mathrm{M}$ sodium citrate

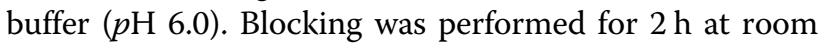
temperature using $2 \%$ horse serum followed by overnight incubation with primary antibody at $4{ }^{\circ} \mathrm{C}$. The corresponding secondary antibody was conjugated to Alexa Fluor 488 or Alexa Fluor 647 (Invitrogen). Sections were incubated with secondary antibodies for $1 \mathrm{~h}$ at room temperature, followed by cell nucleus staining with DAPI for $10 \mathrm{~min}$. Imaging was performed using Olympus IX73 imaging system.

\section{Aortic ring sprouting assay}

To examine the migration and proliferation of SMCs in an ex vivo condition, the mouse aortic ring sprouting assay was performed as described previously ${ }^{48}$. In brief, fresh thoracic aortae were harvested from 12-week old 
$S m p d 1^{+/+}$and $S m p d 1^{-/-}$mice and placed in sterile DMEM buffer. The endothelial layer was removed by the injection of air bubbles. After carefully removing periadventitial fat and connective tissues, the aortae were sliced into ring segments approximately $0.5 \mathrm{~mm}$ in thickness. The aortic rings were rinsed with DMEM medium and then embedded in 24-well plates previously coated with $200 \mu \mathrm{L}$ synthetic basement membrane (Corning ${ }^{\oplus}$ Matrigel $^{\circ}$ ) per well on ice. At least 10-15 rings per group were used for each experiment. The plate was placed at room temperature for $15 \mathrm{~min}$ and then incubated for $1 \mathrm{~h}$ in a humidified incubator $\left(37^{\circ} \mathrm{C}, 5 \% \mathrm{CO}_{2}\right)$. Subsequently, $500 \mu \mathrm{l}$ DMEM culture medium containing $20 \%$ FBS was added into each well. The explants were maintained in a humidified incubator $\left(37^{\circ} \mathrm{C}, 5 \% \mathrm{CO}_{2}\right)$ with medium replaced every other day. For the treatment group, the culture medium was replaced by fresh DMEM containing 20\% FBS and $30 \mathrm{ng} / \mathrm{ml}$ PDGF-BB. After 5 days, the outgrowth of cells was observed and imaged using an inverted microscope (Olympus IX73). Digital images of $S m p d 1^{+/+}$and $S m p d 1^{-/-}$aortic ring sections were analyzed using Image-Pro Plus software. Each outgrowth emerging directly from the ring was identified (number of sprouts), traced, and branch points marked. ImageJ segmented line tool was then used to measure the longest continuous branch, sum length of all branches (total branch length), and furthest distance from the ring for each outgrowth.

\section{Statistics analysis}

Data are presented as mean \pm standard deviation. All in vitro experiments were analyzed by the Student $t$-test or two-way ANOVA with genotype and treatment as category factors, followed by a Bonferroni's multiple comparisons test if applicable. A Students $t$-test was used to detect significant difference between two groups. The statistical analysis was performed by GraphPad Prism 6.0 software (GraphPad Software, USA). $P<0.05$ was considered statistically significant.

\section{Acknowledgements}

This work was supported by the grants from National Institutes of Health (R01HL122769, R01HL122937, R15HL124458) and the Young Scientists Fund of the National Natural Science Foundation of China (Grant No. 81202095).

\section{Author details}

'Department of Oncology, Tongji Hospital, Tongji Medical College, Huazhong University of Science and Technology, Wuhan, China. ${ }^{2}$ Department of Pharmacological and Pharmaceutical Sciences, College of Pharmacy, University of Houston, Houston, USA

\section{Conflict of interest}

The authors declare that they have no conflict of interest.

\section{Publisher's note}

Springer Nature remains neutral with regard to jurisdictional claims in published maps and institutional affiliations.
Received: 6 August 2018 Revised: 29 October 2018 Accepted: 31 October 2018

Published online: 19 November 2018

\section{References}

1. Gomez, D. \& Owens, G. K. Smooth muscle cell phenotypic switching in atherosclerosis. Cardiovasc Res 95, 156-164 (2012).

2. Ronnov-Jessen, L., Petersen, O. W., Koteliansky, V. E. \& Bissell, M. J. The origin of the myofibroblasts in breast cancer. Recapitulation of tumor environment in culture unravels diversity and implicates converted fibroblasts and recruited smooth muscle cells. J. Clin. Invest 95, 859-873 (1995).

3. Hao, H. et al. Phenotypic modulation of intima and media smooth muscle cells in fatal cases of coronary artery lesion. Arterioscler. Thromb. Vasc. Biol. 26, 326-332 (2006).

4. Hinz, B. et al. The myofibroblast: one function, multiple origins. Am. J. Pathol. 170, 1807-1816 (2007).

5. Kendall, R. T. \& Feghali-Bostwick, C. A. Fibroblasts in fibrosis: novel roles and mediators. Front. Pharmacol. 5, 123 (2014).

6. Van Linthout, S., Miteva, K. \& Tschope, C. Crosstalk between fibroblasts and inflammatory cells. Cardiovasc. Res. 102, 258-269 (2014).

7. Zhang, Y., Li, X., Becker, K. A. \& Gulbins, E. Ceramide-enriched membrane domains-structure and function. Biochim. Biophys. Acta 1788, 178-183 (2009).

8. McGovern, M. M. et al. Lipid abnormalities in children with types A and B Niemann Pick disease. J. Pediatr. 145, 77-81 (2004).

9. McGovern, M. M. et al. A prospective, cross-sectional survey study of the natural history of Niemann-Pick disease type B. Pediatrics 122, e341-349 (2008).

10. Leger, A. J. et al. Adeno-associated virus-mediated expression of acid sphingomyelinase decreases atherosclerotic lesion formation in apolipoprotein $\mathrm{E}$ (-/-) mice. J. Gene Med. 13, 324-332 (2011).

11. Leventhal, A. R., Chen, W., Tall, A. R. \& Tabas, I. Acid sphingomyelinase-deficient macrophages have defective cholesterol trafficking and efflux. J. Biol. Chem. 276, 44976-44983 (2001).

12. Liu, J. et al. Macrophage sphingomyelin synthase 2 deficiency decreases atherosclerosis in mice. Circ. Res. 105, 295-303 (2009).

13. Tabas, I., Williams, K. J. \& Boren, J. Subendothelial lipoprotein retention as the initiating process in atherosclerosis: update and therapeutic implications. Circulation 116, 1832-1844 (2007).

14. Oorni, K, Posio, P., Ala-Korpela, M., Jauhiainen, M. \& Kovanen, P. T. Sphingomyelinase induces aggregation and fusion of small very low-density lipoprotein and intermediate-density lipoprotein particles and increases their retention to human arterial proteoglycans. Arterioscler. Thromb. Vasc. Biol. $\mathbf{2 5}$ 1678-1683 (2005).

15. Klionsky, D. J. \& Emr, S. D. Autophagy as a regulated pathway of cellular degradation. Science 290, 1717-1721 (2000).

16. $\mathrm{Xu}, \mathrm{M}$. et al. Regulation of dynein-mediated autophagosomes trafficking by ASM in CASMCs. Front. Biosci. (Landmark Ed.) 21, 696-706 (2016).

17. $L i, X$. et al. Control of autophagy maturation by acid sphingomyelinase in mouse coronary arterial smooth muscle cells: protective role in atherosclerosis. J. Mol. Med (Berl.) 92, 473-485 (2014).

18. Wei, Y. M. et al. Enhancement of autophagy by simvastatin through inhibition of Rac1-mTOR signaling pathway in coronary arterial myocytes. Cell Physiol. Biochem 31, 925-937 (2013).

19. Martin-Garrido, A. et al. Transforming growth factor beta inhibits platelet derived growth factor-induced vascular smooth muscle cell proliferation via Akt-independent, Smad-mediated cyclin D1 downregulation. PLOS ONE 8, e79657 (2013).

20. Wang, Y. C., Cui, X. B., Chuang, Y. H. \& Chen, S. Y. Janus Kinase 3, a Novel Regulator for Smooth Muscle Proliferation and Vascular Remodeling. Arterioscler. Thromb. Vasc. Biol. 37, 1352-1360 (2017).

21. Prockop, D. J. \& Kivirikko, K. I. Collagens: molecular biology, diseases, and potentials for therapy. Annu Rev. Biochem 64, 403-434 (1995).

22. Gelse, K, Poschl, E. \& Aigner, T. Collagens-structure, function, and biosynthesis, Adv. Drug Deliv. Rev. 55, 1531-1546 (2003).

23. Toma, I. \& McCaffrey, T. A. Transforming growth factor-beta and atherosclerosis: interwoven atherogenic and atheroprotective aspects. Cell Tissue Res. 347, 155-175 (2012).

24. Zhang, $\mathrm{H}$. et al. PDGFRs are critical for PI3K/Akt activation and negatively regulated by mTOR. J. Clin. Invest. 117, 730-738 (2007). 
25. Murakami, S. \& Motohashi, H. Roles of Nrf2 in cell proliferation and differentiation. Free Radic. Biol. Med. 88, 168-178 (2015).

26. Bennett, M. R., Sinha, S. \& Owens, G. K. Vascular Smooth Muscle Cells in Atherosclerosis. Circ. Res. 118, 692-702 (2016).

27. Mack, M. \& Yanagita, M. Origin of myofibroblasts and cellular events triggering fibrosis. Kidney Int. 87, 297-307 (2015).

28. Moulton, K. S., et al. PTEN deficiency promotes pathological vascular remodeling of human coronary arteries. JCl Insight. 3, e97228 (2018).

29. Long, X. et al. Smooth muscle calponin: an unconventional CArG-dependent gene that antagonizes neointimal formation. Arterioscler. Thromb. Vasc. Biol. 31, 2172-2180 (2011).

30. Kim, T. R. et al. SM22alpha-induced activation of p16INK4a/retinoblastoma pathway promotes cellular senescence caused by a subclinical dose of gamma-radiation and doxorubicin in HepG2 cells. Biochem. Biophys. Res. Commun. 400, 100-105 (2010).

31. Pathak, S. et al. MiR-155 modulates the inflammatory phenotype of intestinal myofibroblasts by targeting SOCS1 in ulcerative colitis. Exp. Mol. Med. 47, e164 (2015)

32. Lacolley, P., Regnault, V., Nicoletti, A., Li, Z. \& Michel, J. B. The vascular smooth muscle cell in arterial pathology: a cell that can take on multiple roles. Cardiovasc. Res. 95, 194-204 (2012).

33. Dong, N. et al. Autophagy downregulates thrombin-induced VSMCs proliferation through lysosomal pathway. Int. J. Cardiol. 159, 156-158 (2012).

34. Hu, P., Lai, D., Lu, P., Gao, J. \& He, H. ERK and Akt signaling pathways are involved in advanced glycation end product-induced autophagy in rat vascular smooth muscle cells. Int. J. Mol. Med. 29, 613-618 (2012).

35. Salabei, J. K. et al. PDGF-mediated autophagy regulates vascular smooth muscle cell phenotype and resistance to oxidative stress. Biochem. J. 451, 375-388 (2013)

36. Salabei, J. K. \& Hill, B. G. Implications of autophagy for vascular smooth muscle cell function and plasticity. Free Radic. Biol. Med. 65, 693-703 (2013).

37. Kim, J. Y. et al. Apamin inhibits PDGF-BB-induced vascular smooth muscle cell proliferation and migration through suppressions of activated Akt and Erk signaling pathway. Vasc. Pharmacol. 70, 8-14 (2015).
38. Golden, E. B., Pellicciotta, I., Demaria, S., Barcellos-Hoff, M. H. \& Formenti, S. C. The convergence of radiation and immunogenic cell death signaling pathways. Front.Oncol. 2, 88 (2012).

39. Gharibi, B., Ghuman, M. S. \& Hughes, F. J. Akt- and Erk-mediated regulation of proliferation and differentiation during PDGFRbeta-induced MSC self-renewal. J. Cell Mol. Med. 16, 2789-2801 (2012).

40. Andrabi, S., Gjoerup, O. V., Kean, J. A., Roberts, T. M. \& Schaffhausen, B. Protein phosphatase $2 \mathrm{~A}$ regulates life and death decisions via Akt in a contextdependent manner. Proc. Natl Acad. Sci. USA 104, 19011-19016 (2007).

41. Li, G. et al. Enhanced epithelial-to-mesenchymal transition associated with lysosome dysfunction in podocytes: role of p62/Sequestosome 1 as a signaling hub. Cell Physiol. Biochem. 35, 1773-1786 (2015).

42. Nezis, I. P. \& Stenmark, H. p62 at the interface of autophagy, oxidative stress signaling, and cancer. Antioxid. Redox Signal. 17, 786-793 (2012).

43. Moscat, J. \& Diaz-Meco, M. T. p62: a versatile multitasker takes on cancer. Trends Biochem. Sci. 37, 230-236 (2012).

44. Boini, K. M. et al. Acid sphingomyelinase gene deficiency ameliorates the hyperhomocysteinemia-induced glomerular injury in mice. Am. J. Pathol. 179 2210-2219 (2011)

45. Li, X. et al. TRAlL death receptor 4 signaling via lysosome fusion and membrane raft clustering in coronary arterial endothelial cells: evidence from ASM knockout mice. J. Mol. Med. (Berl.) 91, 25-36 (2013).

46. $\mathrm{Xu}, \mathrm{M}$. et al. $\mathrm{NAD}(\mathrm{P}) \mathrm{H}$ oxidase-dependent intracellular and extracellular $\mathrm{O}^{*}{ }^{*}$ production in coronary arterial myocytes from CD38 knockout mice. Free Radic. Biol. Med. 52, 357-365 (2012).

47. Grootaert, M. O. et al. Defective autophagy in vascular smooth muscle cells accelerates senescence and promotes neointima formation and atherogenesis. Autophagy 11, 2014-2032 (2015)

48. Wang, $\mathbf{H}$. et al. Inhibition of Epac1 suppresses mitochondrial fission and reduces neointima formation induced by vascular injury. Sci. Rep. 6, 36552 (2016). 